\title{
Homotopy colimits of classifying spaces of abelian subgroups of a finite group
}

\author{
CiHAN OKaY
}

\begin{abstract}
The classifying space $B G$ of a topological group $G$ can be filtered by a sequence of subspaces $B(q, G), q \geq 2$, using the descending central series of free groups. If $G$ is finite, describing them as homotopy colimits is convenient when applying homotopy theoretic methods. In this paper we introduce natural subspaces $B(q, G)_{p} \subset B(q, G)$ defined for a fixed prime $p$. We show that $B(q, G)$ is stably homotopy equivalent to a wedge of $B(q, G)_{p}$ as $p$ runs over the primes dividing the order of $G$. Colimits of abelian groups play an important role in understanding the homotopy type of these spaces. Extraspecial 2-groups are key examples, for which these colimits turn out to be finite. We prove that for extraspecial 2-groups of order $2^{2 n+1}, n \geq 2, B(2, G)$ does not have the homotopy type of a $K(\pi, 1)$ space, thus answering in a negative way a question posed by Adem in [1]. For a finite group $G$, we compute the complex $K$-theory of $B(2, G)$ modulo torsion.
\end{abstract}

55R10; 55N15, 55Q52

\section{Introduction}

In [1], Adem introduced a natural filtration

$$
B(2, G) \subset \cdots \subset B(q, G) \subset \cdots \subset B(\infty, G)=B G
$$

of the classifying space $B G$ of a topological group $G$. For a fixed $q \geq 2$, let $\Gamma^{q}\left(F_{n}\right)$ denote the $q^{\text {th }}$ stage of the descending central series of the free group on $n$ generators. Then $B(q, G)$ is the geometric realization of the simplicial space whose $n$-simplices are the spaces of homomorphisms $\operatorname{Hom}\left(F_{n} / \Gamma^{q}\left(F_{n}\right), G\right)$. In this article we study homotopy-theoretic properties of these spaces.

Let $G$ be a finite group and $p$ a prime dividing the order of $G$. Consider the free pro- $p$ group $P_{n}$, the pro- $p$ completion of the free group $F_{n}$. As noted in [1], the geometric realization of the simplicial set $\mathbf{n} \mapsto \operatorname{Hom}\left(P_{n} / \Gamma^{q}\left(P_{n}\right), G\right)$ gives a natural subspace of $B(q, G)$, and is denoted by $B(q, G)_{p}$. We prove that there is a stable homotopy equivalence: 
Theorem 1.1 Suppose that $G$ is a finite group. There is a natural weak equivalence

$$
\bigvee_{p|| G \mid} \Sigma B(q, G)_{p} \rightarrow \Sigma B(q, G) \quad \text { for all } q \geq 2
$$

induced by the inclusions $B(q, G)_{p} \rightarrow B(q, G)$.

Let $\mathcal{N}(q, G)$ denote the collection of subgroups of nilpotency class less than $q$ and $G(q)=\operatorname{colim}_{\mathcal{N}(q, G)} A$. The key observation in [1] is the following fibration

$$
\operatorname{hocolim}_{\mathcal{N}(q, G)} G(q) / A \longrightarrow B(q, G) \longrightarrow B G(q),
$$

which can be constructed using the (homotopy) colimit description of $B(q, G)$. This raises the following question of whether they are actually homotopy equivalent, posed in [1].

Question 1.2 (Adem [1, page 15]) If $G$ is a finite group, are the spaces $B(q, G)$ Eilenberg-Mac Lane spaces of type $K(\pi, 1)$ ?

One of the objectives of this paper is to show the existence of a certain class of groups for which $B(2, G)$ does not have the homotopy type of a $K(\pi, 1)$ space. The following theorem is used to show that extraspecial 2 -groups are examples of such groups. (At a given order there are two types up to isomorphism.)

Theorem 1.3 Let $G_{n}$ denote an extraspecial 2-group of order $2^{2 n+1}$ then

$$
\pi_{1}\left(B\left(2, G_{n}\right)\right) \cong \operatorname{colim}_{\mathcal{N}\left(2, G_{n}\right)} A \cong G_{n} \times \mathbb{Z} / 2 \quad \text { for } n \geq 2 .
$$

Indeed for the central products $D_{8} \circ D_{8}$ and $D_{8} \circ Q_{8}$, that is $n=2$, the higher homotopy groups are given by

$$
\pi_{i}\left(B\left(2, G_{2}\right)\right) \cong \pi_{i}\left(\bigvee^{151} S^{2}\right) \text { for } i>1 .
$$

Another natural question is to compute the complex $K$-theory of a homotopy colimit. The main tool to study a representable generalized cohomology theory of a homotopy colimit is the Bousfield-Kan spectral sequence whose $E_{2}$-term consists of the derived functors of the inverse limit functor. We address this problem for the homotopy colimit of classifying spaces of abelian subgroups of a finite group, that is, for $B(2, G)$.

Theorem 1.4 There is an isomorphism

$$
\mathbb{Q} \otimes K^{i}(B(2, G)) \cong \begin{cases}\mathbb{Q} \oplus \bigoplus_{p \| G \mid} \mathbb{Q}_{p}^{n_{p}} & \text { if } i=0, \\ 0 & \text { if } i=1,\end{cases}
$$

where $n_{p}$ is the number of (non-identity) elements of order a power of $p$ in $G$. 
Torsion groups can appear in $K^{1}(B(2, G))$. An explicit example is the case of $G_{2}$, there is an isomorphism

$$
K^{i}\left(B\left(2, G_{2}\right)\right) \cong \begin{cases}\mathbb{Z} \oplus \mathbb{Z}_{2}^{31} & \text { if } i=0, \\ (\mathbb{Z} / 2)^{9} & \text { if } i=1 .\end{cases}
$$

The organization of this paper is as follows. In Section 2.1 we introduce the spaces $B(q, G)$ and $B(q, G)_{p}$ for discrete groups and list some of their basic properties. In Section 3 we assume $G$ is finite, and describe these spaces as homotopy colimits. We prove a stable decomposition of $B(q, G)$ (Theorem 1.1). In Section 4 we turn to the higher limits as a preparation for the complex $K$-theory of $B(2, G)$. Under some assumptions, we prove a vanishing result of certain higher limits (Theorem 4.7). The main result of Section 5 is the computation of $\mathbb{Q} \otimes K^{*}(B(2, G))$ as given in Theorem 1.4. Section 6 contains a general observation on colimits of abelian groups (Theorem 6.1). As an application, in Section 7 we compute the colimit of abelian subgroups of an extraspecial 2-group (Theorem 1.3). Key examples which illustrate the main features discussed throughout the paper are given in Section 8. Basic properties of homotopy colimits are explained in the appendix, Appendix A.

Acknowledgements The author would like to thank A Adem, for his supervision; J Smith, for pointing out Proposition 4.5; F Cohen and J M Gómez, for commenting on an early version of this paper.

\section{Filtrations of classifying spaces}

\subsection{Preliminaries}

Let $\Delta$ be the category whose objects are finite non-empty totally ordered sets $\mathbf{n}, n \geq 0$, with $n+1$ elements

$$
0 \longrightarrow 1 \longrightarrow 2 \longrightarrow \cdots \longrightarrow n
$$

and whose morphisms $\theta: \mathbf{m} \rightarrow \mathbf{n}$ are order preserving set maps or alternatively functors. The nerve of a small category $\mathbf{C}$ is the simplicial set

$$
B \mathbf{C}_{n}=\text { Hom }_{\text {Cat }}(\mathbf{n}, \mathbf{C}) \text {. }
$$

For instance, the geometric realization of the nerve $B G_{\bullet}$ of a discrete group $G$ (regarded as a category with one object) is the classifying space $B G$. An equivalent way of describing the classifying space is as follows. The assignment

$$
\mathbf{n}=\left(0 \stackrel{e_{1}}{\longrightarrow} 1 \stackrel{e_{2}}{\longrightarrow} \cdots \stackrel{e_{n}}{\longrightarrow} n\right) \mapsto F_{n},
$$


where $F_{n}$ is the free group generated by $\left\{e_{1}, e_{2}, \ldots, e_{n}\right\}$, defines a faithful functor $\Delta \rightarrow$ Grp injective on objects. Then the classifying space of $G$ is isomorphic to the simplicial set

$$
B G_{\bullet}: \Delta^{\mathrm{op}} \rightarrow \text { Set; } \quad \mathbf{n} \mapsto \operatorname{Hom}_{\mathbf{G r p}}\left(F_{n}, G\right) .
$$

A generalization of this construction to certain quotients of free groups is studied in [1]. We recall the constructions and describe some alternative versions.

Definition 2.1 For a group $Q$ define a chain of groups inductively: $\Gamma^{1}(Q)=Q$, $\Gamma^{q+1}(Q)=\left[\Gamma^{q}(Q), Q\right]$. The descending central series of $Q$ is the normal series

$$
1 \subset \cdots \subset \Gamma^{q+1}(Q) \subset \Gamma^{q}(Q) \subset \cdots \subset \Gamma^{2}(Q) \subset \Gamma^{1}(Q)=Q .
$$

Now take $Q$ to be the free group $F_{n}$. For $q>0$, the natural maps $F_{n} \rightarrow F_{n} / \Gamma^{q}\left(F_{n}\right)$ induce inclusions of sets

$$
\operatorname{Hom}_{\mathbf{G r p}}\left(F_{n} / \Gamma^{q}\left(F_{n}\right), G\right) \subset \operatorname{Hom}_{\mathbf{G r p}}\left(F_{n}, G\right) .
$$

Furthermore, the simplicial structure of $B G \bullet$ induces a simplicial structure on the collection of these subsets.

Definition 2.2 Let $G$ be a discrete group. We define a sequence of simplicial sets $B \bullet(q, G), q \geq 2$, by the assignment

$$
\mathbf{n} \mapsto \operatorname{Hom}_{\mathbf{G r p}}\left(F_{n} / \Gamma^{q}\left(F_{n}\right), G\right)
$$

and denote the geometric realization $|B(q, G) \bullet|$ by $B(q, G)$. Define $B \bullet(\infty, G)=B G \bullet$ and $B(\infty, G)=B G$ by convention.

As in [1] this gives rise to a filtration

$$
B(2, G) \subset B(3, G) \subset \cdots \subset B(q, G) \subset B(q+1, G) \subset \cdots \subset B(\infty, G)=B G
$$

of the classifying space of $G$.

\section{$2.2 p$-local version}

An alternative construction is obtained by replacing the free group $F_{n}$ by its pro- $p$ completion, namely the free pro- $p$ group $P_{n}$; see Dixon, du Sautoy, Mann and Segal [5] for their basic properties. It can be defined using the $p$-descending central series of the free group

$$
P_{n}=\lim _{\longleftarrow} F_{n} / \Gamma_{p}^{q}\left(F_{n}\right)
$$


where $\Gamma_{p}^{q}\left(F_{n}\right)$ are defined recursively:

$$
\Gamma_{p}^{1}\left(F_{n}\right)=F_{n} \text { and } \Gamma_{p}^{q+1}\left(F_{n}\right)=\left[\Gamma_{p}^{q}\left(F_{n}\right), F_{n}\right]\left(\Gamma_{p}^{q}\left(F_{n}\right)\right)^{p}
$$

The group $P_{n}$ contains $F_{n}$ as a dense subgroup. Observe that each quotient $F_{n} / \Gamma_{p}^{q}\left(F_{n}\right)$ is a finite $p$-group.

Let TGrp denote the category of topological groups. We consider continuous group homomorphisms $\phi \in \operatorname{Hom}_{\mathbf{T G r p}}\left(P_{n}, G\right)$ where $G$ is a discrete group. The image of $\phi$ is a finite $p$-subgroup of $G$. This follows from the fact that $P_{n}$ is compact and if $K$ is a subgroup of $P_{n}$ of finite index then $\left|P_{n}: K\right|$ is a power of $p$ by [5, Lemma 1.18]. Then there exists $q \geq 1$ such that $\phi$ factors as:

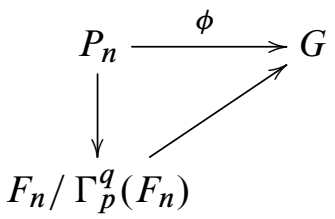

This implies that $\operatorname{Hom}_{\mathbf{T G r p}}\left(P_{n}, G\right) \subset \operatorname{Hom}_{\mathbf{G r p}}\left(F_{n}, G\right)$ and also there is a simplicial structure induced from $B G_{\bullet}$.

We remark that a theorem of Serre on topological groups says that any (abstract) group homomorphism from a finitely generated pro- $p$ group to a finite group is continuous [5, Theorem 1.17]. This implies that when $G$ is finite any (abstract) homomorphism $\phi: P_{n} \rightarrow G$ is continuous. More generally, as a consequence of Nikolov and Segal [16, Theorem 1.13], if every subgroup of $G$ is finitely generated then the image of an abstract homomorphism $\phi$ is a finite group, hence in this case $\phi$ is also continuous.

Definition 2.3 Let $p$ be a prime integer and $G$ a discrete group. We define a sequence of simplicial sets $B_{\bullet}(q, G)_{p}, q \geq 2$, by

$$
\mathbf{n} \mapsto \operatorname{Hom}_{\mathbf{T G r p}}\left(P_{n} / \Gamma^{q}\left(P_{n}\right), G\right) .
$$

Define $B_{\bullet}(\infty, G)_{p}$ to be the simplicial set $\mathbf{n} \mapsto \operatorname{Hom}_{\mathbf{T G r p}}\left(P_{n}, G\right)$ and set $B(\infty, G)_{p}=$ $\left|B_{\bullet}(\infty, G)_{p}\right|$.

Let us examine the simplicial set $B_{\bullet}(\infty, G)_{p}$. There is a natural map

$$
\theta: \lim _{q} \operatorname{Hom}\left(F_{n} / \Gamma_{p}^{q}\left(F_{n}\right), G\right) \rightarrow \operatorname{Hom}\left(P_{n}, G\right)
$$

induced by the projections $P_{n} \rightarrow F_{n} / \Gamma_{p}^{q}\left(F_{n}\right)$. This map is injective since it is induced by injective maps and surjective since $\phi: P_{n} \rightarrow G$ factors through $F_{n} / \Gamma_{p}^{q}\left(F_{n}\right) \rightarrow G$ for some $q \geq 1$, as observed above. Thus $\theta$ is a bijection of sets. Colimits in the category 
of simplicial sets can be constructed dimension-wise and the geometric realization functor commutes with colimits. Therefore, for a discrete group $G$, we note that there is a homeomorphism

$$
\lim _{q} B(q, G, p) \rightarrow B(\infty, G)_{p}
$$

induced by $\theta$, where $B(q, G, p)$ is the geometric realization of the simplicial set defined by $B_{n}(q, G, p)=\operatorname{Hom}_{\mathbf{G r p}}\left(F_{n} / \Gamma_{p}^{q}\left(F_{n}\right), G\right)$.

\section{The case of finite groups}

We restrict our attention to finite groups. Note that the following collections of subgroups have an initial object (the trivial subgroup), and they are closed under taking subgroups and the conjugation action of $G$. Define a collection of subgroups for $q \geq 2$ by

$$
\mathcal{N}(q, G)=\left\{H \subset G \mid \Gamma^{q}(H)=1\right\} .
$$

These are nilpotent subgroups of $G$ of class less than $q$. Observe that $\mathcal{N}(2, G)$ is the collection of abelian subgroups of $G$.

Denote the poset of $p$-subgroups of $G$ by $\mathcal{S}_{p}(G)$ (including the trivial subgroup) and set

$$
\mathcal{N}(q, G)_{p}=\mathcal{S}_{p}(G) \cap \mathcal{N}(q, G) .
$$

When $q=2$ this is the collection of abelian $p$-subgroups of $G$.

\subsection{Homotopy colimits}

In [1] Adem observes that there is a homeomorphism

$$
\operatorname{colim}_{\mathcal{N}(q, G)} B A \rightarrow B(q, G)
$$

and furthermore the natural map

$$
\operatorname{hocolim}_{\mathcal{N}(q, G)} B A \rightarrow \operatorname{colim}_{\mathcal{N}(q, G)} B A
$$

turns out to be a weak equivalence. A similar statement holds for the spaces $B(q, G)_{p}$.

Proposition 3.1 Suppose that $G$ is a finite group. Then there is a homeomorphism

$$
B(q, G)_{p} \cong \operatorname{colim}_{\mathcal{N}(q, G)_{p}} B P
$$

and the natural map

$$
\operatorname{hocolim}_{\mathcal{N}(q, G)_{p}} B P \rightarrow \operatorname{colim}_{\mathcal{N}(q, G)_{p}} B P
$$

is a weak equivalence. 
Proof There is a natural bijection of sets

$$
\operatorname{colim}_{\mathcal{N}(q, G)_{p}} \operatorname{Hom}_{\mathbf{G r p}}\left(F_{n}, P\right) \rightarrow \operatorname{Hom}_{\mathbf{G r p}}\left(P_{n} / \Gamma^{q}\left(P_{n}\right), G\right) .
$$

The image of a morphism $P_{n} / \Gamma^{q}\left(P_{n}\right) \rightarrow G$ is a $p$-group of nilpotency class at most $q$. Conversely, if $P \subset G$ is a $p$-group of nilpotency class $q$ then $F_{n} \rightarrow P$ induces a map $P_{n} \rightarrow P$ by taking the pro- $p$ completions, and factors through the quotient $P_{n} / \Gamma^{q}\left(P_{n}\right)$. This induces the desired homeomorphism

$$
B(q, G)_{p} \cong \operatorname{colim}_{\mathcal{N}(q, G)_{p}} B P .
$$

The natural map from the homotopy colimit to the ordinary colimit is a weak equivalence since the diagram of spaces is free (see Appendix A).

Remark 3.2 For the homotopy colimits considered in this paper, it is sufficient to consider the sub-poset determined by the intersections of the maximal objects of the relevant poset. More precisely, let $M_{1}, M_{2}, \ldots, M_{k}$ denote the maximal groups in the poset $\mathcal{N}(q, G)$ and $\mathcal{M}(q, G)=\left\{\bigcap_{J} M_{i} \mid J \subset\{1,2, \ldots, k\}\right\}$. The inclusion map

$$
\mathcal{M}(q, G) \rightarrow \mathcal{N}(q, G)
$$

is right cofinal and hence induces a weak equivalence (see Welker, Ziegler and Živaljević [21, Proposition 3.10])

$$
\operatorname{hocolim}_{\mathcal{M}(q, G)} B M \rightarrow \operatorname{hocolim}_{\mathcal{N}(q, G)} B A .
$$

A similar observation holds for $\mathcal{N}(q, G)_{p}$.

\subsection{A stable decomposition of $B(q, G)$}

Recall that a finite nilpotent group $N$ is a direct product of its Sylow $p$-subgroups:

$$
N \cong \prod_{p|| N \mid} N_{(p)}
$$

For a prime $p$ dividing the order of the group $G$, and $N \in \mathcal{N}(q, G)$, define

$$
\pi_{p}(N)= \begin{cases}N_{(p)} & \text { if } p|| N \mid \\ 1 & \text { otherwise }\end{cases}
$$

Lemma 3.3 The inclusion map $\iota_{p}: \mathcal{N}(q, G)_{p} \rightarrow \mathcal{N}(q, G)$ of posets induces a weak equivalence

$$
\operatorname{hocolim}_{\mathcal{N}(q, G)_{p}} B P \rightarrow \operatorname{hocolim}_{\mathcal{N}(q, G)} B \pi_{p}(A) .
$$


Proof By freeness (Appendix A) it is enough to consider ordinary colimits. Let $B: \mathcal{N}(q, G)_{p} \rightarrow$ Top denote the functor $P \mapsto B P$. The map $\pi_{p}: \mathcal{N}(q, G) \rightarrow \mathcal{N}(q, G)_{p}$ defined by $A \mapsto \pi_{p}(A)$ induces an inverse to the map induced by $\iota_{p}$ on the colimit

$$
\operatorname{colim}_{\mathcal{N}(q, G)_{p}} B P \rightarrow \operatorname{colim}_{\mathcal{N}(q, G)} B \pi_{p}(A) .
$$

This follows from the fact that $\pi_{p} \circ \iota_{p}$ is the identity on $\mathcal{N}(q, G)_{p}$.

Theorem 3.4 Suppose that $G$ is a finite group. There is a natural weak equivalence

$$
\bigvee_{p|| G \mid} \Sigma B(q, G)_{p} \rightarrow \Sigma B(q, G) \quad \text { for all } q \geq 2
$$

induced by the inclusions $B(q, G)_{p} \rightarrow B(q, G)$.

Proof First note that the nerve of the poset $\mathcal{N}(q, G)$ is contractible since the trivial subgroup is an initial object. Note that for $A \in \mathcal{N}(q, G)$ each $B A$ is pointed via the inclusion $B 1 \rightarrow B A$.

Let $\mathcal{J}$ denote the pushout category $0 \leftarrow 01 \rightarrow 1$. Define a functor $F$ from the product category $\mathcal{J} \times \mathcal{N}(q, G)$ to Top by

$$
F((0, A))=\mathrm{pt}, \quad F((01, A))=B A \quad \text { and } \quad F((1, A))=\mathrm{pt} .
$$

Commutativity of homotopy colimits implies that

$$
\operatorname{hocolim}_{\mathcal{J}} \operatorname{hocolim}_{\mathcal{N}(q, G)} F \cong \operatorname{hocolim}_{\mathcal{J} \times \mathcal{N}(q, G)} F \cong \operatorname{hocolim}_{\mathcal{N}(q, G)} \operatorname{hocolim}_{\mathcal{J}} F .
$$

The nerve of $\mathcal{N}(q, G)$ is contractible, in which case we can identify the homotopy colimit over $\mathcal{J}$ as the suspension and conclude that the map

$$
\Sigma\left(\operatorname{hocolim}_{\mathcal{N}(q, G)} B A\right) \rightarrow \operatorname{hocolim}_{\mathcal{N}(q, G)} \Sigma(B A)
$$

is a homeomorphism. The natural map induced by the suspension of the inclusions $B A_{(p)} \rightarrow B A$,

$$
\bigvee_{p \| A \mid} \Sigma B A_{(p)} \rightarrow \Sigma B A
$$

is a weak equivalence. This follows from the splitting of suspension of products,

$$
\Sigma\left(B A_{(p)} \times B A_{(q)}\right) \simeq \Sigma\left(B A_{(p)}\right) \vee \Sigma\left(B A_{(q)}\right) \vee \Sigma\left(B A_{(p)} \wedge B A_{(q)}\right),
$$

and from the equivalence $\Sigma\left(B A_{(p)} \wedge B A_{(q)}\right) \simeq$ pt when $p$ and $q$ are coprime. The latter follows from the Künneth theorem and the Hurewicz theorem by considering the homology isomorphism induced by the inclusion $B A_{(p)} \vee B A_{(q)} \rightarrow B A_{(p)} \times B A_{(q)}$. 
Invariance of homotopy colimits under natural transformations which induce a weak equivalence on each object, the homeomorphism (3.4.1) and Lemma 3.3 give the weak equivalences

$$
\begin{aligned}
\Sigma\left(\operatorname{hocolim}_{\mathcal{N}(q, G)} B A\right) & \cong \operatorname{hocolim}_{\mathcal{N}(q, G)} \Sigma(B A) \\
& \simeq \operatorname{hocolim}_{\mathcal{N}(q, G)} \bigvee_{p \| A \mid} \Sigma\left(B A_{(p)}\right) \\
& \simeq \bigvee_{p \| G \mid} \Sigma\left(\operatorname{hocolim}_{\mathcal{N}(q, G)} B \pi_{p}(A)\right) \\
& \simeq \bigvee_{p \| G \mid} \Sigma\left(\operatorname{hocolim}_{\mathcal{N}(q, G)_{p}} B P\right) .
\end{aligned}
$$

When commuting the wedge product with the homotopy colimit, again an argument using the commutativity of homotopy colimits can be used similar to the suspension case. Note that the wedge product is a homotopy colimit.

This stable equivalence immediately implies the following decomposition for a generalized cohomology theory.

Theorem 3.5 There is an isomorphism

$$
\tilde{h}^{*}(B(q, G)) \cong \prod_{p|| G \mid} \tilde{h}^{*}\left(B(q, G)_{p}\right) \quad \text { for all } q \geq 2,
$$

where $\widetilde{h}^{*}$ denotes a reduced cohomology theory.

Therefore one can study homological properties of $B(q, G)$ at a fixed prime. In particular, this theorem applies to the complex $K$-theory of $B(q, G)$ and each piece corresponding to $B(q, G)_{p}$ can be computed using the Bousfield-Kan spectral sequence [3].

\subsection{Homotopy types of $B(q, G)$ and $B(q, G)_{p}$}

We follow the discussion on the fundamental group of $B(q, G)$ from Adem [1], similar properties are satisfied by $B(q, G)_{p}$. Let $\mathcal{N}$ be a collection of subgroups of a finite group $G$. We require that $\mathcal{N}$ has an initial element and is closed under taking subgroups. In particular, it can be taken as $\mathcal{N}(q, G)$ or $\mathcal{N}(q, G)_{p}$. The fundamental group of a homotopy colimit is described by Farjoun [9, Corollary 5.1]. There is an isomorphism

$$
\pi_{1}\left(\operatorname{hocolim}_{\mathcal{N}} B A\right) \cong \operatorname{colim}_{\mathcal{N}} A
$$


where the colimit is in the category of groups. We will study colimits of (abelian) groups in the next section in more detail.

For each $A \in \mathcal{N}$, there is a natural fibration $G / A \rightarrow B A \rightarrow B G$ and taking homotopy colimits one obtains a fibration

$$
\operatorname{hocolim}_{\mathcal{N}} G / A \rightarrow \operatorname{hocolim}_{\mathcal{N}} B A \rightarrow B G \text {. }
$$

This is a consequence of a theorem of Puppe (see Farjoun [8, Appendix HL]). The point is that each fibration has the same base space $B G$. Associated to this fibration there is an exact sequence of homotopy groups

$$
\begin{aligned}
1 \longrightarrow \pi_{1}\left(\operatorname{hocolim}_{\mathcal{N}} G / A\right) \longrightarrow & \pi_{1}\left(\operatorname{hocolim}_{\mathcal{N}} B A\right) \\
& \stackrel{\psi}{\longrightarrow} \pi_{1}(B G) \longrightarrow \pi_{0}\left(\operatorname{hocolim}_{\mathcal{N}} G / A\right) \longrightarrow 0,
\end{aligned}
$$

where $\psi$ is the natural map

$$
\pi_{1}\left(\operatorname{hocolim}_{\mathcal{N}} B A\right) \cong \operatorname{colim}_{\mathcal{N}} A \rightarrow G
$$

induced by the inclusions $A \rightarrow G$. Note that if $\mathcal{N}$ contains all cyclic subgroups of $G$ then hocolim $_{\mathcal{N}} G / A$ is connected. Since in this case, the commutativity of

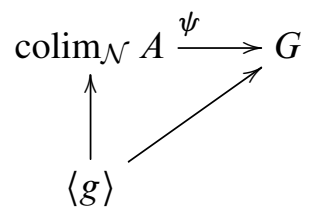

implies that $\psi$ is surjective.

We point out here that for $\mathcal{N}=\mathcal{N}(q, G)$ the homotopy fibre hocolim $\mathcal{N}_{\mathcal{N}} G / A$ is homotopy equivalent to the pull-back of the universal principal $G$-bundle

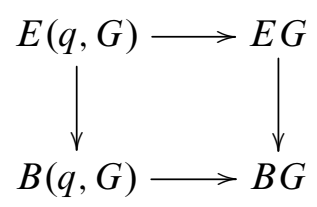

and this pull-back $E(q, G)$ can be defined as the geometric realization of a simplicial set as described by Adem [1]. It can be identified as a colimit

$$
E(q, G) \cong \operatorname{colim}_{\mathcal{N}(q, G)} G \times_{A} E A
$$


and there is a commutative diagram

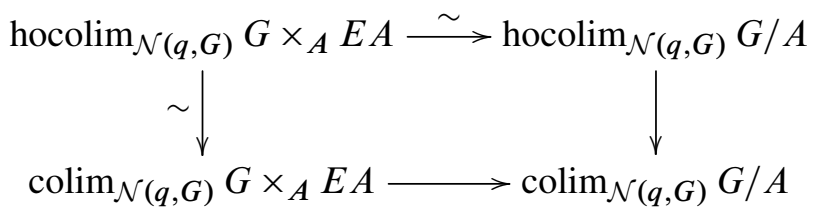

induced by the contractions $E A \rightarrow \mathrm{pt}$, and the weak equivalences as indicated. The exact sequence (3.5.2) becomes

$$
0 \longrightarrow T(q) \longrightarrow G(q) \stackrel{\psi}{\longrightarrow} G \longrightarrow 0,
$$

where $G(q)=\pi_{1}(B(q, G))$ and $T(q)=\pi_{1}(E(q, G))$.

Consider the universal cover $\widetilde{B}(q, G)$ of $B(q, G)$. Again using the theorem of Puppe, it can be described as the homotopy fibre of the homotopy colimit of the natural fibrations $G(q) / A \longrightarrow B A \longrightarrow B G(q)$ (see Adem [1, Theorem 4.4])

$$
\widetilde{B}(q, G) \simeq \operatorname{hocolim}_{\mathcal{N}(q, G)} G(q) / A \longrightarrow B(q, G)
$$

The question of $B(q, G)$ having the homotopy type of a $K(\pi, 1)$ space is equivalent to asking whether the classifying space functor $B$ commutes with colimits

$$
B(q, G)=\operatorname{colim}_{\mathcal{N}(q, G)} B A \rightarrow B\left(\operatorname{colim}_{\mathcal{N}(q, G)} A\right) .
$$

The difference is measured by the simply connected, finite-dimensional complex $\widetilde{B}(q, G)$, or equivalently by the values of the higher limits

$$
H^{i}(\widetilde{B}(q, G) ; \mathbb{Z}) \cong \lim _{\mathcal{N}(q, G)}^{i} \mathbb{Z}[G(q) / A]
$$

as implied by the Bousfield-Kan spectral sequence.

The class of extraspecial 2-groups provides examples for which $B(2, G)$ is not homotopy equivalent to a $K(\pi, 1)$ space. This follows from the computation of the fundamental group of $B(2, G)$ that is the colimit of abelian subgroups of $G$; see Section 7 and Section 8.

\section{Higher limits}

As the higher limits appear in the $E_{2}$-term of the Bousfield-Kan spectral sequence, in this section we discuss some of their properties. The main theorem of this section is a 
vanishing result of the higher limits of the contravariant part of a pre-Mackey functor $R: \mathbf{d n} \rightarrow \mathbf{A b}$ where $\mathbf{d n}$ denotes the poset of non-empty subsets of $\{1, \ldots, n\}$ ordered by reverse inclusion.

Let $\mathbf{C}$ be a small category and $F: \mathbf{C} \rightarrow \mathbf{A b}$ be a contravariant functor from $\mathbf{C}$ to the category of abelian groups. $\mathbf{A b}^{\mathbf{C}}$ denotes the category of contravariant functors $\mathbf{C} \rightarrow \mathbf{A b}$. Observe that

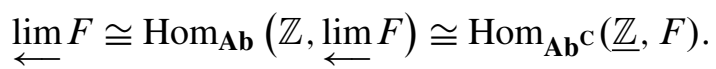

Definition 4.1 Derived functors of the inverse limit of $F: \mathbf{C} \rightarrow \mathbf{A b}$ are defined by

$$
\lim ^{i} F \equiv \operatorname{Ext}_{\mathbf{A b}}^{i} \mathbf{c}(\underline{\mathbb{Z}}, F)
$$

A projective resolution of $\underline{\mathbb{Z}}$ in $\mathbf{A} \mathbf{b}^{\mathbf{C}}$ can be obtained in the following way, for details see Webb [20]. First note that the functors $F_{c}: \mathbf{C} \rightarrow \mathbf{A b}$ defined by $F_{c}\left(c^{\prime}\right)=\mathbb{Z} \operatorname{Hom}_{\mathbf{C}}\left(c^{\prime}, c\right)$ are projective functors and by Yoneda's Lemma,

$$
\operatorname{Hom}_{\mathbf{A b}} \mathbf{c}\left(F_{c}, F\right) \cong F(c) \text {. }
$$

Let $\mathbf{C} \backslash-: \mathbf{C} \rightarrow \mathbf{S}$ denote the functor which sends an object $c$ of $\mathbf{C}$ to the nerve of the under category $\mathbf{C} \backslash c$. The nerve $B(\mathbf{C} \backslash c)$ is contractible since the object $c \rightarrow c$ is initial. Then we define a resolution $P_{*} \rightarrow \underline{\mathbb{Z}}$ of the constant functor as the composition $P_{*}=C_{*} \circ \mathbf{C} \backslash-$, where $C_{*}: \mathbf{S} \rightarrow \mathbf{A b}$ is the functor which sends a simplicial set $X$ to the associated chain complex $C_{*}(X)=\mathbb{Z}\left[X_{*}\right]$. At each degree $n$, there are isomorphisms

$$
\operatorname{Hom}_{\mathbf{A b}^{\mathbf{C}}}\left(P_{n}, F\right) \cong \operatorname{Hom}_{\mathbf{A} \mathbf{b}^{\mathbf{C}}}\left(\bigoplus_{x_{0} \leftarrow \ldots \leftarrow x_{n}} F_{x_{n}}, F\right) \cong \prod_{x_{0} \leftarrow x_{1} \leftarrow \ldots \leftarrow x_{n}} F\left(x_{n}\right) .
$$

Differentials are induced by the simplicial maps between chains of morphisms. The following result describes them explicitly.

Lemma 4.2 (Oliver [17]) $\lim ^{i} F \cong H^{i}\left(C^{*}(\mathbf{C} ; F), \delta\right)$, where

$$
C^{n}(\mathbf{C} ; F)=\prod_{x_{0} \leftarrow x_{1} \leftarrow \ldots \leftarrow x_{n}} F\left(x_{n}\right)
$$

for all $n \geq 0$ and where for $U \in C^{n}(\mathbf{C} ; F)$,

$\delta(U)\left(x_{0} \longleftarrow x_{1} \longleftarrow \cdots \longleftarrow x_{n} \stackrel{\phi}{\longleftarrow} x_{n+1}\right)$

$=\sum_{i=0}^{n}(-1)^{i} U\left(x_{0} \leftarrow \cdots \leftarrow \hat{x}_{i} \leftarrow \cdots \leftarrow x_{n+1}\right)+(-1)^{n+1} F(\phi)\left(U\left(x_{0} \leftarrow \cdots \leftarrow x_{n}\right)\right)$. 
A useful property of higher limits, which allows the change of the indexing category, is the following.

Proposition 4.3 (Jackowski and McClure [13, Lemma 3.1]) Fix a small category $\mathbf{C}$ and a contravariant functor $F: \mathbf{C} \rightarrow \mathbf{A b}$. Let $\mathbf{D}$ be a small category and assume that $g: \mathbf{D} \rightarrow \mathbf{C}$ has a left adjoint. Set $g^{*} F=F \circ g: \mathbf{D} \rightarrow \mathbf{A b}$ then $H^{*}(\mathbf{C} ; F) \cong$ $H^{*}\left(\mathbf{D} ; g^{*} F\right)$.

Assume that the indexing category $\mathbf{C}$ is a finite partially ordered set (poset) whose morphisms are $i \rightarrow j$ whenever $i \leq j$. We consider an appropriate filtration of $F$ as in Grodal [11].

Definition 4.4 A height function on a poset is a strictly increasing map of posets $h t: \mathbf{C} \rightarrow \mathbb{Z}$.

A convenient height function can be defined by $h t(A)=-\operatorname{dim}\left(\left|\mathbf{C}_{\geq A}\right|\right)$, where $\mathbf{C}_{\geq A}$ is the subposet of $\mathbf{C}$ consisting of elements $C \geq A$. Let $N=\operatorname{dim}(|\mathbf{C}|)$ then it is immediate that $\lim ^{i} F$ vanishes for $i>N$. The functor $F$ can be filtered in such a way that the associated quotient functors are concentrated at a single height. Define a sequence of subfunctors

$$
F_{N} \subset \cdots \subset F_{2} \subset F_{1} \subset F_{0}
$$

by $F_{i}(A)=0$ if $h t(A)>-i$ and $F_{i}(A)=F(A)$ otherwise. This induces a decreasing filtration on the cochain complexes hence there is an associated spectral sequence whose $E_{0}$-term is

$$
E_{0}^{i, j}=C^{i+j}\left(\mathbf{C} ; F_{i} / F_{i+1}\right)=\prod_{A \in \mathbf{C} \mid h t(A)=-i} \operatorname{Hom}_{\mathbb{Z}}\left(C_{i+j}\left(\left|\mathbf{C}_{\geq A}\right|,\left|\mathbf{C}_{>A}\right|\right), F(A)\right)
$$

with differentials $d_{0}: C^{k}\left(\mathbf{C} ; F_{i} / F_{i+1}\right) \rightarrow C^{k+1}\left(\mathbf{C} ; F_{i} / F_{i+1}\right) . E_{1}$-term is the cohomology of the pair $\left(\left|\mathbf{C}_{\geq A}\right|,\left|\mathbf{C}_{>A}\right|\right)$ in coefficients $F(A)$ that is

$$
E_{1}^{i, j}=\prod_{A \in \mathbf{C} \mid h t(A)=-i} H^{i+j}\left(\left(\left|\mathbf{C}_{\geq A}\right|,\left|\mathbf{C}_{>A}\right|\right) ; F(A)\right)
$$

Fix $A$ and let $A^{\prime}>A$ such that $h t\left(A^{\prime}\right)=h t(A)+1$, then differential $d_{1}$ can be described as the composition

$$
\begin{aligned}
H^{k}\left(\left(\left|\mathbf{C}_{\geq A^{\prime}}\right|,\left|\mathbf{C}_{>A^{\prime}}\right|\right) ; F\left(A^{\prime}\right)\right) \stackrel{F\left(A \leq A^{\prime}\right)}{\longrightarrow} H^{k}\left(\left(\left|\mathbf{C}_{>A}\right|, \mid \mathbf{C}_{>A, h t \geq h t(A)+2 \mid) ; F(A))}\right.\right. \\
\stackrel{\partial}{\longrightarrow} H^{k+1}\left(\left(\left|\mathbf{C}_{\geq A}\right|,\left|\mathbf{C}_{>A}\right|\right) ; F(A)\right)
\end{aligned}
$$


where $\partial$ is the boundary map associated to the triple

$$
\left(\left|\mathbf{C}_{\geq A}\right|,\left|\mathbf{C}_{>A}\right|,\left|\mathbf{C}_{>A, h t \geq h t(A)+2}\right|\right) .
$$

We now consider higher limits over specific kinds of diagrams. Let $\mathbf{d n}$ be the category associated to the non-degenerate simplexes of the standard $n$-simplex, the objects are increasing sequences of numbers $\sigma_{k}=\left[i_{0}<\cdots<i_{k}\right]$, where $0 \leq i_{j} \leq n$ and the morphisms are generated by the face maps $d_{j}\left(\left[i_{0}<\cdots<i_{k}\right]\right)=\left[i_{0}<\cdots<\hat{i}_{j}<\cdots<i_{k}\right]$ for $0 \leq j \leq k$.

Proposition 4.5 For any contravariant functor $F: \mathbf{d n} \rightarrow \mathbf{A b}$ the spectral sequence (4.4.1) collapses onto the horizontal axis hence gives a long exact sequence

$$
0 \longrightarrow \prod_{\sigma_{0}} F\left(\sigma_{0}\right) \longrightarrow \prod_{\sigma_{1}} F\left(\sigma_{1}\right) \longrightarrow \cdots \longrightarrow \prod_{\sigma_{n-1}} F\left(\sigma_{n-1}\right) \longrightarrow F\left(\sigma_{n}\right) \longrightarrow 0
$$

and where for $U \in C_{\mathbf{d n}}^{k-1}(F)=\prod_{\sigma_{k-1}} F\left(\sigma_{k-1}\right)$,

$$
\delta^{k-1}(U)\left(\sigma_{k}\right)=\sum_{j=0}^{k}(-1)^{k-j} F\left(d_{j}\right) U\left(d_{j}\left(\sigma_{k}\right)\right) .
$$

Proof For a simplex $\sigma_{k} \in \mathbf{d n}$, let $\mathbf{d n}_{\geq k}$ be the poset of simplices $\sigma \geq \sigma_{k}$ and $\mathbf{d n}_{>k}$ denote the poset of simplices $\sigma>\sigma_{k}$. Observe that the pair $\left(\left|\mathbf{d n}_{\geq k}\right|,\left|\mathbf{d} \mathbf{n}_{>k}\right|\right)$ is homeomorphic to $\left(\left|\Delta^{k}\right|,\left|\partial \Delta^{k}\right|\right)$, where $\Delta^{k}$ is the standard $k$-simplex with boundary $\partial \Delta^{k}$. The spectral sequence of the filtration has

$$
E_{1}^{k, j}=\prod_{\sigma_{k}} H^{k+j}\left(\left(\left|\mathbf{d n}_{\geq k}\right|,\left|\mathbf{d n}_{>k}\right|\right) ; F\left(\sigma_{k}\right)\right) .
$$

Therefore $E_{1}$-term vanishes unless $j=0$, and otherwise

$$
E_{1}^{k, 0}=\prod_{\sigma_{k}} H^{k}\left(\left(\left|\mathbf{d n}_{\geq k}\right|,\left|\mathbf{d n}_{>k}\right|\right) ; F\left(\sigma_{k}\right)\right)=\prod_{\sigma_{k}} F\left(\sigma_{k}\right)
$$

collapses onto the horizontal axes, resulting in a long exact sequence. The differential is induced by the alternating sum of the face maps $d_{j}\left(\sigma_{k}\right)$ for $0 \geq j \geq k$.

In some cases, it is possible to show that the higher limits vanish. The next theorem is an illustration of this instance. First let us recall the definition of a pre-Mackey functor in the sense of Dress [6]. Let $M: \mathbf{C} \rightarrow \mathbf{D}$ be a bifunctor, that is a pair of functors $\left(M^{*}, M_{*}\right)$ such that $M^{*}$ is contravariant, $M_{*}$ is covariant and both coincide on objects. 
Definition 4.6 A pre-Mackey functor is a bifunctor $M: \mathbf{C} \rightarrow \mathbf{D}$ such that for any pull-back diagram

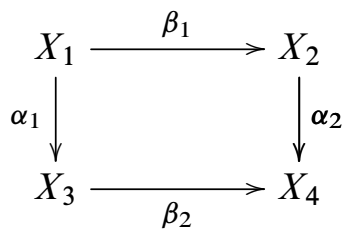

in $\mathbf{C}$ the diagram

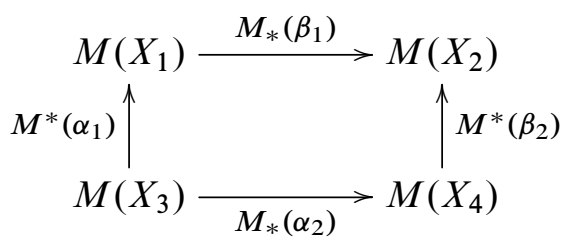

commutes.

We give a direct proof of the following theorem, which can also be deduced from an application of Jackowski-McClure [13, Theorem 5.15] to the category $\mathbf{d n}$.

Theorem 4.7 Let $R: \mathbf{d n} \rightarrow \mathbf{A b}$ be a pre-Mackey functor then $\lim ^{s} R^{*}=0$ for $s>0$.

Proof We do induction on $n$. The case $n=0$ is trivial since the complex (4.5.1) is concentrated at degree 0 . There are inclusions of categories

$$
\iota_{0}: \mathbf{d n}-\mathbf{1} \rightarrow \mathbf{d n}
$$

defined by $\left[i_{1}<\cdots<i_{k}\right] \mapsto\left[i_{1}+1<\cdots<i_{k}+1\right]$ and

$$
\iota_{1}: \mathbf{d n}-\mathbf{1} \rightarrow \mathbf{d n},
$$

where $\left[i_{1}<\cdots<i_{k}\right] \mapsto\left[0<i_{1}+1<\cdots<i_{k}+1\right]$. Let $C_{\mathbf{d n}}^{*}$ denote the complex $C_{\mathbf{d n}}^{*}(R)$ in (4.5.1) that is $C_{\mathbf{d n}}^{k}=\prod_{\sigma_{k}} R\left(\sigma_{k}\right)$. Define a filtration $C_{-1}^{*} \subset C_{0}^{*} \subset C_{\mathbf{d n}}^{*}$ such that

$$
C_{0}^{k}=\prod_{\sigma_{k}=\left[0<i_{1}<\cdots<i_{k}\right]} R\left(\sigma_{k}\right)
$$

and $C_{-1}^{k}=C_{0}^{k}$ for $k>0$ and $C_{-1}^{0}=0$. Then by induction

$$
H^{i}\left(C_{\mathbf{d n}}^{*} / C_{0}^{*}\right)=H^{i}\left(C_{\mathbf{d n}-\mathbf{1}}^{*}\left(R \circ \iota_{0}\right)\right)=0
$$

for $i>0$ and

$$
H^{i}\left(C_{-1}^{*}\right)=H^{i-1}\left(C_{\mathbf{d n}-\mathbf{1}}^{*}\left(R \circ \iota_{1}\right)\right)=0
$$


for $i>1$. And short exact sequences associated to the filtration $C_{-1}^{*} \subset C_{0}^{*} \subset C_{\mathbf{d n}}^{*}$ implies that it suffices to prove $H^{1}\left(C_{0}^{*}\right)=0$.

For a simplex $\sigma=[0<i<\cdots<j]$ and morphisms $\alpha_{i}: \sigma \rightarrow[0<i]$ and $\alpha_{j}: \sigma \rightarrow[0<j]$ in $\mathbf{d n}$, set $\phi_{\sigma}=R_{*}\left(\alpha_{j}\right) R^{*}\left(\alpha_{i}\right)$.

For a fixed $i$, define a map $\Phi_{i}: R([0<i]) \rightarrow R([0])$ by

$$
\Phi_{i}=\sum_{\sigma=[0<i<\cdots<j]}(-1)^{|\sigma|-1} R_{*}\left(d_{1}^{j}\right) \phi_{\sigma}, \quad \text { where } d_{1}^{j}:[0<j] \rightarrow[0],
$$

where $|\sigma|$ denotes the dimension of the simplex $\sigma$. Combining these maps for all $1 \leq i \leq n$ define $\Phi: \prod_{l=1}^{n} R([0<l]) \rightarrow R([0])$ by

$$
\Phi=\sum_{i=1}^{n} \Phi_{i} \pi_{i}
$$

where $\pi_{i}$ denotes the natural projection $\prod_{l=1}^{n} R([0<l]) \rightarrow R([0<i])$ to the $i^{\text {th }}$ factor.

We need to show that if $U$ is in the kernel of $\delta^{1}$, then $\delta^{0} \Phi(U)=U$ or equivalently $R^{*}\left(d_{1}^{i}\right) \Phi(U)=\pi_{i}(U)$ for all $1 \leq i \leq n$. Fix $k$, and compute the composition

$$
\begin{aligned}
R^{*}\left(d_{1}^{k}\right) \Phi & =\sum_{i=1}^{n} \sum_{\sigma=[0<i<\cdots<j]}(-1)^{|\sigma|-1} R^{*}\left(d_{1}^{k}\right) R_{*}\left(d_{1}^{j}\right) \phi_{\sigma} \\
& =\pi_{k}+\sum_{i=1}^{n}\left(\sum_{\sigma \mid k \in \sigma \neq[0<k]}(-1)^{|\sigma|-1} R^{*}\left(d_{1}^{k}\right) R_{*}\left(d_{1}^{j}\right) \phi_{\sigma}\right. \\
& \left.+\sum_{\sigma \mid k \notin \sigma}(-1)^{|\sigma|-1} R^{*}\left(d_{1}^{k}\right) R_{*}\left(d_{1}^{j}\right) \phi_{\sigma}\right) .
\end{aligned}
$$

In the second line first use the identity $R^{*}\left(d_{1}^{k}\right) R_{*}\left(d_{1}^{j}\right) \phi_{[0<k]}=\pi_{k}$ then separate the summation into two parts such that it runs over the simplices which contain $\{k\}$ and do not contain $\{k\}$. Suppose that $\sigma=[0<i<\cdots<j]$ does not contain $\{k\}$ and $\bar{\sigma}$ denote the simplex obtained from $\sigma$ by adjoining $\{k\}$. There are three possibilities for $\bar{\sigma}$ :

$$
\bar{\sigma}= \begin{cases}{[0<i<\cdots<k<\cdots<j]} & \text { if } i<k<j \\ {[0<i<\cdots<j<k]} & \text { if } j<k \\ {[0<k<i<\cdots<j]} & \text { if } k<i\end{cases}
$$


The corresponding terms of the first two cases cancel out when $\sigma$ and $\bar{\sigma}$ are paired off in the summation, since the diagram

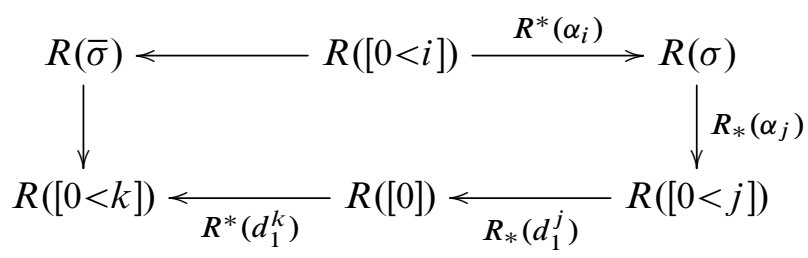

commutes, and the corresponding terms become equal with opposite signs. So the equation simplifies to

$$
R^{*}\left(d_{1}^{k}\right) \Phi=\pi_{k}+\sum_{\sigma=[0<k<i<\cdots<j]}(-1)^{|\sigma|-1}\left(R^{*}\left(d_{1}^{k}\right) R_{*}\left(d_{1}^{j}\right) \phi_{\sigma}-R^{*}\left(d_{1}^{k}\right) R_{*}\left(d_{1}^{j}\right) \phi_{d_{1} \sigma}\right)
$$

where the sum runs over the simplices starting with $\{0<k\}$. The maps

$$
\sigma \stackrel{\theta}{\longrightarrow}[0<k<i] \text { and } \sigma \stackrel{\vartheta}{\longrightarrow}[0<k]
$$

and also the two face maps

$$
[0<i] \stackrel{d_{1}}{\longleftarrow}[0<k<i] \stackrel{d_{2}}{\longrightarrow}[0<k]
$$

in our indexing category $\mathbf{d n}$ give a commutative diagram

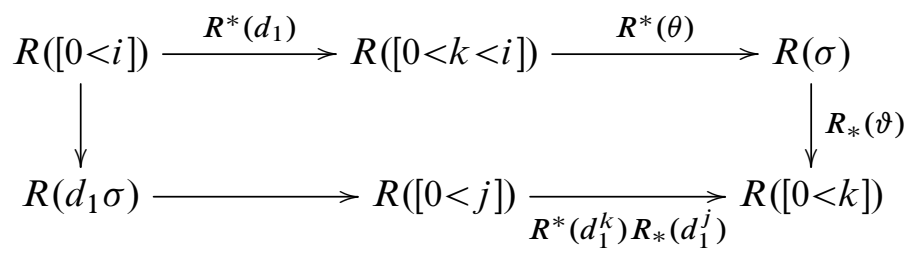

Hence we can write

$$
R^{*}\left(d_{1}^{k}\right) \Phi=\pi_{k}+\sum_{\sigma=[0<k<i<\cdots<j]}(-1)^{|\sigma|-1} R_{*}(\vartheta) R^{*}(\theta)\left(R^{*}\left(d_{2}\right)-R^{*}\left(d_{1}\right)\right) .
$$

Now suppose that $U$ is in the kernel of $\delta^{1}: C_{0}^{1} \rightarrow C_{0}^{2}$, the summation above vanishes since $\left(R^{*}\left(d_{2}\right)-R^{*}\left(d_{1}\right)\right)(U)=0$. Therefore

$$
R^{*}\left(d_{1}^{k}\right) \Phi(U)=\pi_{k}(U),
$$

which implies that $H^{1}\left(C_{0}^{*}\right)=0$. 


\subsection{Higher limits of the representation ring functor}

Let $G$ be a finite group and $p$ be a prime dividing its order. Let $M_{0}, \ldots, M_{n}$ denote the maximal abelian $p$-subgroups of $G$. Consider the contravariant functor $R: \mathbf{d n} \rightarrow \mathbf{A b}$ defined by $\sigma \mapsto R\left(M_{\sigma}\right)$, where $M_{\sigma}=\bigcap_{i \in \sigma} M_{i}$ and $R(H)$ is the Grothendieck ring of complex representations of $H$. Note that $\mathbb{Q} \otimes R$ is a pre-Mackey functor: If $\alpha: \sigma \rightarrow \sigma^{\prime}$ set $A=\bigcap_{i \in \sigma} M_{i}$ and $B=\bigcap_{i \in \sigma^{\prime}} M_{i}$ then

$$
\left(\mathbb{Q} \otimes R^{*}(\alpha), \mathbb{Q} \otimes R_{*}(\alpha)\right)=\left(\operatorname{res}_{B, A}, \frac{1}{|B: A|} \operatorname{ind}_{A, B}\right) .
$$

Hence Theorem 4.7 implies the following.

Corollary 4.8 If $R: \mathbf{d n} \rightarrow \mathbf{A b}$ is defined as above then $\lim ^{s} \mathbb{Q} \otimes R^{*}=0$ for $s>0$.

Proof We give an alternative proof that is specific to the representation ring functor. Let $U: \mathbf{G r p} \rightarrow$ Set denote the forgetful functor which sends a group to the underlying set. The isomorphism $\left.\mathbb{C} \otimes R\left(M_{\sigma}\right) \cong \operatorname{Hom}_{\text {Set }}\left(M_{\sigma}, \mathbb{C}\right)=H^{0}\left(U\left(M_{\sigma}\right) ; \mathbb{C}\right)\right)$ induces an isomorphism

$$
{\underset{\lim }{\longleftarrow}}^{s} \mathbb{C} \otimes R\left(M_{\sigma}\right) \cong \lim ^{s} H^{0}\left(U\left(M_{\sigma}\right) ; \mathbb{C}\right)
$$

Note that $U\left(M_{\sigma}\right)$ is a finite set of points. Consider the space hocolim $\mathbf{d n} U\left(M_{\sigma}\right)$ and the associated cohomology spectral sequence

$$
E_{2}^{s, t}=\lim _{\mathbf{d n}}^{s} H^{t}\left(U\left(M_{\sigma}\right) ; \mathbb{C}\right) \Rightarrow H^{s+t}\left(\operatorname{hocolim}_{\mathbf{d n}} U\left(M_{\sigma}\right) ; \mathbb{C}\right) .
$$

Now, the diagram $\mathbf{d n} \rightarrow$ Top defined by $\sigma \mapsto U\left(M_{\sigma}\right)$ is free (Appendix A), consists of inclusions of finite sets. Therefore the natural map

$$
\operatorname{hocolim}_{\mathbf{d n}} U\left(M_{\sigma}\right) \rightarrow \operatorname{colim}_{\mathbf{d n}} U\left(M_{\sigma}\right)=U(G)
$$

is a homotopy equivalence, note that the latter is a finite set. The spectral sequence collapses at $E_{2}$-page and gives

$$
\lim ^{s} H^{0}\left(U\left(M_{\sigma}\right) ; \mathbb{C}\right) \cong H^{s}\left(\operatorname{hocolim}_{\mathbf{d n}} U\left(M_{\sigma}\right) ; \mathbb{C}\right),
$$

which vanishes for $s>0$.

\section{$5 \quad K$-theory of $B(2, G)$}

In this section, we assume that $G$ is a finite group. We study the complex $K$-theory of $B(q, G)$ when $q=2$. Theorem 3.5 gives a decomposition

$$
\tilde{K}^{*}(B(2, G)) \cong \bigoplus_{p|| G \mid} \tilde{K}^{*}\left(B(2, G)_{p}\right)
$$


of the reduced $K$-theory of $B(2, G)$. For each such prime $p$ recall the fibration

$$
\operatorname{hocolim}_{\mathcal{N}(q, G)_{p}} G / P \longrightarrow B(q, G)_{p} \longrightarrow B G
$$

from Section 3. Furthermore, the Borel construction commutes with homotopy colimits and gives a weak equivalence

$$
B(q, G)_{p} \simeq\left(\operatorname{hocolim}_{\mathcal{N}(q, G)_{p}} G / P\right) \times_{G} E G .
$$

There is the Atiyah-Segal completion theorem [2] which relates the equivariant $K-$ theory of a $G$-space $X$ to the complex $K$-theory of its Borel construction $X \times{ }_{G} E G$.

Equivariant $\boldsymbol{K}$-theory Complex equivariant $K$-theory is a $\mathbb{Z} / 2$-graded cohomology theory defined for compact spaces using $G$-equivariant complex vector bundles (see Segal [18]). Recall that

$$
K_{G}^{n}(G / H) \cong \begin{cases}R(H) & \text { if } n=0, \\ 0 & \text { if } n=1,\end{cases}
$$

where $H \subseteq G$ and recall that $R(H)$ denotes the Grothendieck ring of complex representations of $H$. Note that in particular, $K_{G}^{0}(\mathrm{pt}) \cong R(G)$ and $K_{G}^{*}(X)$ is an $R(G)$-module via the natural map $X \rightarrow$ pt.

Let $X$ be a compact $G$-space and $I(G)$ denote the kernel of the augmentation map $R(G) \stackrel{\varepsilon}{\longrightarrow} \mathbb{Z}$. The Atiyah-Segal completion theorem [2] states that $K^{*}\left(X \times_{G} E G\right)$ is the completion of $K_{G}^{*}(X)$ at the augmentation ideal $I(G)$. In particular, taking $X$ to be a point this theorem implies that

$$
K^{n}(B G) \cong \begin{cases}R(G)^{\wedge} & n=0, \\ 0 & n=1 .\end{cases}
$$

The completion $R(G)^{\wedge}$ can be described using the restriction maps to a Sylow $p-$ subgroup for each $p$ dividing the order of the group $G$. Let $I_{p}(G)$ denote the quotient of $I(G)$ by the kernel of the restriction map $I(G) \rightarrow I\left(G_{(p)}\right)$ to a Sylow $p$-subgroup. There are isomorphisms of abelian groups

$$
K^{n}(B G) \cong \begin{cases}\mathbb{Z} \oplus \bigoplus_{p|| G \mid} \mathbb{Z}_{p} \otimes I_{p}(G) & n=0 \\ 0 & n=1 ;\end{cases}
$$

see Lück [15]. Note that if $G$ is a nilpotent group, equivalently $G \cong \prod_{p|| G \mid} G_{(p)}$, the restriction map $I(G) \rightarrow I\left(G_{(p)}\right)$ is surjective and

$$
\widetilde{K}^{0}(B G) \cong I(G)^{\wedge} \cong \bigoplus_{p|| G \mid} \mathbb{Z}_{p} \otimes I\left(G_{(p)}\right)
$$


We also use the fact that the completion of $R(H)$ with respect to $I(H)$ is isomorphic to the completion with respect to $I(G)$ as an $R(G)$-module via the restriction map $R(G) \rightarrow R(H)$.

\section{$5.1 \quad K$-theory of $B(2, G)$}

The $E_{2}$-page of the equivariant version of the Bousfield-Kan spectral sequence (see Lee [14]) for the equivariant $K$-theory of $\operatorname{hocolim}_{\mathcal{N}(q, G)_{p}} G / P$ is given by

$$
E_{2}^{s, t} \cong \lim _{\mathcal{N}(q, G)_{p}}^{s} K_{G}^{t}(G / P) \cong \begin{cases}\lim _{\mathcal{N}(q, G)_{p}}^{s} R(P) & \text { if } t \text { is even }, \\ 0 & \text { if } t \text { is odd },\end{cases}
$$

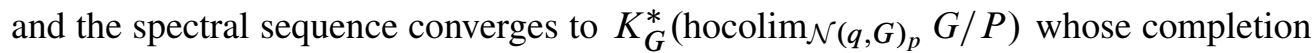
is $K^{*}\left(\operatorname{hocolim}_{\mathcal{N}(q, G)_{p}} B P\right)$. We will split off the trivial part of $E_{2}^{s, t}$ and consider

$$
\widetilde{E}_{2}^{s, t} \cong \lim _{\mathcal{N}(q, G)_{p}}^{s} I(P) \quad \text { for } t \text { even. }
$$

Note that the splitting $R(P) \cong \mathbb{Z} \oplus I(P)$ gives

$$
\lim _{\mathcal{N}(q, G)_{p}}^{s} R(P) \cong \lim _{\mathcal{N}(q, G)_{p}}^{s} I(P)
$$

for $s>0$ since the nerve of $\mathcal{N}(q, G)_{p}$ is contractible. The following result describes the terms of this spectral sequence for the case $q=2$.

Lemma 5.1 Let $R: \mathcal{N}(2, G)_{p} \rightarrow$ Ab be the representation ring functor defined by $P \mapsto R(P)$ then $\lim ^{s} R$ are torsion groups for $s>0$. Furthermore $\lim ^{0} R \cong \mathbb{Z}^{n_{p}+1}$, where $n_{p}$ is the number of (non-identity) elements of order a power $\overleftarrow{\text { of } p}$ in $G$.

Proof Let $M_{i}$ for $0 \leq i \leq n$ denote the maximal abelian $p$-subgroups of $G$. Define a functor $g: \mathbf{d n} \rightarrow \mathcal{N}(2, G)_{p}$ by sending a simplex $\left[i_{0}<i_{1}<\cdots<i_{k}\right]$ to the intersection $\bigcap_{j=0}^{k} M_{i_{j}}$. Then $g$ has a left adjoint, namely the functor $P \mapsto \bigcap_{M_{i}: P \subset M_{i}} M_{i}$ sending an abelian $p$-subgroup to the intersection of maximal abelian $p$-subgroups containing it. By Proposition 4.3 we can replace the indexing category $\mathcal{N}(2, G)_{p}$ by $\mathbf{d n}$ and calculate the higher limits over the category $\mathbf{d n}$. Then the first part of the theorem follows from Corollary 4.8 .

For the second part note that $\lim ^{0} R=\lim R$ is a submodule of a free $\mathbb{Z}$-module, hence it is free. Then it suffices to calculate its rank. Tensoring with $\mathbb{C}$ gives an isomorphism

$$
\mathbb{C} \otimes{\underset{\lim }{\longleftarrow}}_{\mathcal{N}(2, G)_{p}}^{0} R(P) \cong \operatorname{Hom}_{\text {Set }}\left(\bigcup_{\mathcal{N}(2, G)_{p}} P, \mathbb{C}\right) .
$$


Recall that $\mathcal{N}(2, G)_{p}$ is the collection of abelian $p$-subgroups of $G$. The result follows from the identity $\bigcup_{\mathcal{N}(2, G)_{p}} P=n_{p}+1$, where $n_{p}$ is the number of (non-identity) elements of order a power of $p$ in $G$.

Theorem 5.2 There is an isomorphism

$$
\mathbb{Q} \otimes K^{i}(B(2, G)) \cong \begin{cases}\mathbb{Q} \oplus \bigoplus_{p|| G \mid} \mathbb{Q}_{p}^{n_{p}} & \text { if } i=0, \\ 0 & \text { if } i=1,\end{cases}
$$

where $n_{p}$ is the number of (non-identity) elements of order a power of $p$ in $G$.

Proof By the decomposition in Theorem 3.5 we work at a fixed prime $p$ dividing the order of $G$. Then by the Atiyah-Segal completion theorem, $K^{*}\left(B(2, G)_{p}\right)$ is isomorphic to the completion of $K_{G}^{*}\left(\operatorname{hocolim}_{\mathcal{N}(2, G)_{p}} G / P\right)$ at the augmentation ideal $I(G)$. Since $R(G)$ is a Noetherian ring the completion $-\otimes_{R(G)} R(G)^{\wedge}$ is exact on finitely generated $R(G)$-modules, hence commutes with taking homology. Moreover, (5.0.1) gives

$$
R(P) \otimes_{R(G)} R(G)^{\wedge} \cong R(P)^{\wedge} \cong \mathbb{Z} \oplus I(P) \otimes \mathbb{Z}_{p}
$$

This isomorphism induces

$$
\widetilde{E}_{r}^{s, t} \otimes_{I(G)} I(G)^{\wedge} \cong \widetilde{E}_{r}^{s, t} \otimes_{\mathbb{Z}} \mathbb{Z}_{p} \quad \text { for } r \geq 2,
$$

which gives an isomorphism between the abutments. For $t$ even and $s>0, \widetilde{E}_{2}^{s, t}$ are torsion by Lemma 5.1. After tensoring with $\mathbb{Q}$, the spectral sequence collapses onto the vertical axis at the $E_{2}$-page and $\mathbb{Q} \otimes \widetilde{K}^{0}\left(B(2, G)_{p}\right) \cong \mathbb{Q} \otimes \mathbb{Z}_{p}^{n_{p}}$, whereas $\mathbb{Q} \otimes K^{1}\left(B(2, G)_{p}\right)$ vanishes.

\section{Colimits of abelian groups}

The fundamental groups of $B(2, G)$ and $B(2, G)_{p}$ are isomorphic to the colimits of the collection of abelian subgroups and abelian $p$-subgroups of $G$, respectively. Therefore it is natural to study the colimit of a collection of abelian groups in greater generality to determine the homotopy properties of these spaces.

Let $\mathcal{A}$ denote a collection of abelian groups closed under taking subgroups. We consider the colimit of the groups in $\mathcal{A}$. This can be constructed as a quotient of the free product $\amalg$ of the groups in the collection

$$
\operatorname{colim}_{\mathcal{A}} A \cong\left(\coprod_{A \in \mathcal{A}} A\right) / \sim
$$


by the normal subgroup generated by the relations $b \sim f(b)$, where $b \in B$ and $f: B \rightarrow A$ runs over the morphisms in $\mathcal{A}$. Let $T$ be a finite abelian group, define the number

$$
d(T)=\sum_{p|| T \mid} \operatorname{rank}\left(T_{(p)}\right)
$$

where $T_{(p)}$ denotes the Sylow $p$-subgroup of $T$. For $r>0$ and a collection of finite abelian groups $\mathcal{A}$, define a sub-collection

$$
\mathcal{A}_{r}=\{A \in \mathcal{A} \mid d(A) \leq r\} .
$$

The main result of this section is the following isomorphism of colimits, which reduces the collection $\mathcal{A}$ to the sub-collection $\mathcal{A}_{2}$. Note that if $\mathcal{A}$ consists of elementary abelian $p$-groups then $\mathcal{A}_{2}$ is the sub-collection of groups of rank at most 2.

Theorem 6.1 The natural map

$$
\operatorname{colim}_{\mathcal{A}_{2}} A \rightarrow \operatorname{colim}_{\mathcal{A}} A
$$

induced by the inclusion map $\mathcal{A}_{2} \rightarrow \mathcal{A}$ is an isomorphism.

The proof follows from the homotopy properties of a complex constructed from the cosets of proper subgroups of a group. We review some background first.

The coset poset Consider the poset $\{x H \mid H \subsetneq G\}$ consisting of cosets of proper subgroups of $G$ ordered by inclusion. The associated complex is denoted by $\mathcal{C}(G)$ and it is studied in Brown [4]. This complex can be identified as a homotopy colimit

$$
\mathcal{C}(G) \simeq \operatorname{hocolim}_{\{H \subsetneq G\}} G / H .
$$

The identification follows from the description of this homotopy colimit as the nerve of the transport category of the poset $\{H \subsetneq G\}$ which is precisely the nerve of the poset $\{x H \mid H \subsetneq G\}$.

When $G$ is solvable, $\mathcal{C}(G)$ has the homotopy type of a bouquet of spheres. To make this statement precise we need a definition from group theory.

Definition 6.2 A chief series of a group $G$ is a series of normal subgroups

$$
1=N_{0} \subset N_{1} \subset \cdots \subset N_{k}=G
$$

for which each factor $N_{i+1} / N_{i}$ is a minimal (nontrivial) normal subgroup of $G / N_{i}$. 
Proposition 6.3 (Brown [4, Proposition 11]) Suppose that $G$ is a solvable finite group and

$$
1=N_{0} \subset N_{1} \subset \cdots \subset N_{k}=G
$$

be a chief series then

$$
\mathcal{C}(G) \simeq \bigvee^{n} S^{d-1}
$$

for some $n>0$, where $d$ is the number of indices $i=1,2, \ldots, k$ such that $N_{i} / N_{i-1}$ has a complement in $G / N_{i-1}$.

We are interested only in the dimensions of the spheres.

Coset poset of abelian groups The number $d$ in Proposition 6.3 for an abelian group $T$ is given by $d(T)$ as introduced at the beginning of this section:

$$
d(T)=\sum_{p \| T \mid} d\left(T_{(p)}\right)=\sum_{p \| T \mid} \operatorname{rank}\left(T_{(p)}\right),
$$

where $T_{(p)}$ denotes the Sylow $p$-subgroup of $T$.

For a finite group $G$, we denote the collection of proper abelian subgroups by $\mathcal{A}(G)$. (It is equal to $\mathcal{N}(2, G)$ when $G$ is non-abelian.)

Proposition 6.4 Let $T$ be a finite abelian group. There is a fibration

$$
\bigvee^{n} S^{d(T)-1} \longrightarrow \operatorname{hocolim}_{\mathcal{A}(T)} B A \longrightarrow B T
$$

for some $n>0$.

Proof The fibre is the coset poset

$$
\mathcal{C}(T) \simeq \operatorname{hocolim}_{\mathcal{A}(T)} T / A
$$

and it is homotopy equivalent to $\bigvee^{n} S^{d(T)-1}$ by Proposition 6.3.

The exact sequence of homotopy groups associated to the fibration (6.4.1) implies the following.

Corollary 6.5 When $d(T) \geq 3$, the natural map

$$
\beta: \operatorname{colim}_{\mathcal{A}(T)} A \rightarrow T
$$

is an isomorphism. 
Proof of Theorem 6.1 We have a sequence of inclusions

$$
\mathcal{A}_{2} \longrightarrow \mathcal{A}_{3} \longrightarrow \cdots \longrightarrow \mathcal{A}_{i} \longrightarrow \mathcal{A}_{i+1} \longrightarrow \cdots \longrightarrow \mathcal{A}_{r}=\mathcal{A}
$$

for some $r>2$. For each $2 \leq i<r$, one can filter these sets further as follows

$$
\mathcal{A}_{i}=\mathcal{A}_{i, 0} \subset \mathcal{A}_{i, 1} \subset \cdots \subset \mathcal{A}_{i, j} \subset \cdots \subset \mathcal{A}_{i, t_{i}}=\mathcal{A}_{i+1},
$$

where $\mathcal{A}_{i, j}=\left\{B \in \mathcal{A}_{i+1} \mid \mathcal{A}(B) \subseteq \mathcal{A}_{i, j-1}\right\}$ for $0<j \leq t_{i}$.

For $2 \leq i<r$ and $0 \leq j<t_{i}$, each of the maps

$$
\operatorname{colim}_{\mathcal{A}_{i, j}} A \longrightarrow \operatorname{colim}_{\mathcal{A}_{i, j+1}} A
$$

has an inverse induced by the compositions: Let $Q \in \mathcal{A}_{i, j+1}-\mathcal{A}_{i, j}$

$$
Q \stackrel{\beta^{-1}}{\longrightarrow} \operatorname{colim}_{\mathcal{A}(Q)} A \longrightarrow \operatorname{colim}_{\mathcal{A}_{i, j}} A,
$$

where $\beta$ is the map in Corollary 6.5 and the second map is induced by the inclusion $\mathcal{A}(Q) \rightarrow \mathcal{A}_{i, j}$. Hence each inclusion map $\mathcal{A}_{i, j} \rightarrow \mathcal{A}_{i, j+1}$ induces an isomorphism on the colimits.

\section{Colimit of abelian subgroups of extraspecial 2-groups}

The colimit of abelian subgroups of a finite group usually turns out to be an infinite group. Simplest examples are amalgamations of the maximal abelian subgroups along the center of the group; see Section 8 for such examples of groups. The smallest example which diverges from this pattern is the extraspecial groups of order 32 . In this section, we consider colimits of abelian subgroups of extraspecial 2-groups and prove that it is a finite group.

An extraspecial 2-group of order $2^{2 n+1}$ fits into an extension of the form

$$
\mathbb{Z} / 2 \longrightarrow G_{n} \stackrel{\pi}{\longrightarrow}(\mathbb{Z} / 2)^{2 n},
$$

where $\mathbb{Z} / 2$ is the center $Z\left(G_{n}\right)$. There are two, up to isomorphism, extraspecial 2-groups $G_{n}^{+}$and $G_{n}^{-}$for a fixed $n$. The first one is isomorphic to a central product of $n$ copies of $D_{8}$ the dihedral group of order 8 , for the second one replace one copy of $D_{8}$ by $Q_{8}$ the quaternion group of order 8 .

We want to compute the colimit of abelian subgroups of $G_{n}$ and we denote the collection of abelian subgroups by $\mathcal{A}\left(G_{n}\right)$. Recall that there is a natural surjective map

$$
\psi: \operatorname{colim}_{\mathcal{A}\left(G_{n}\right)} A \rightarrow G_{n}
$$


induced by the inclusions $A \rightarrow G_{n}$.

In the case of $G_{1}$, which is either $Q_{8}$ or $D_{8}$, the colimit is an amalgamated product of the maximal abelian subgroups along the center. We consider the case $n \geq 2$. There is an exact sequence

$$
1 \longrightarrow Z\left(G_{n}\right) \longrightarrow \operatorname{colim}_{\mathcal{A}\left(G_{n}\right)} A \longrightarrow \operatorname{colim}_{\mathcal{A}\left(G_{n}\right)} A / Z\left(G_{n}\right) \longrightarrow 1
$$

and a commutative diagram

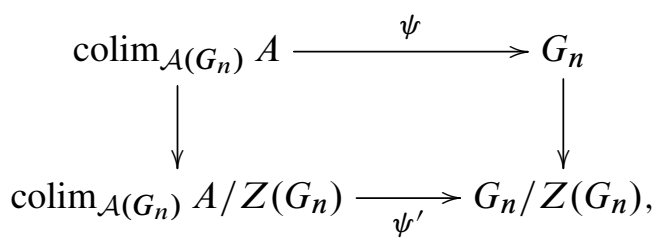

where $\psi$ and $\psi^{\prime}$ are surjective.

It is easier to study $\operatorname{colim}_{\mathcal{A}\left(G_{n}\right)} A / Z\left(G_{n}\right)$, since we can identify this group as a colimit over the polar space of $S p_{2 n}(2)$; see for example Smith [19] for a discussion of polar spaces. Let us explain this identification.

Polar spaces $\operatorname{Regard}(\mathbb{Z} / 2)^{2 n}$ as a vector space over $\mathbb{Z} / 2=\{0,1\}$ and denote it by $V^{2 n}$. There is a non-degenerate symplectic bilinear form $V^{2 n} \times V^{2 n} \rightarrow \mathbb{Z} / 2$ defined by

$$
(v, w)=\left[\pi^{-1}(v), \pi^{-1}(w)\right],
$$

where $[-,-]$ denotes the commutator of given two elements in $G_{n}$. A subgroup $A$ of $G$ is abelian if and only if $\pi(A)$ is an isotropic subspace of $V^{2 n}$ with respect to the bilinear form $(-,-)$, that is $\pi(A) \subset \pi(A)^{\perp}$. One can order these isotropic subspaces by inclusion.

Definition 7.1 The polar space of $S p_{2 n}(2)$ is the poset of (non-trivial) isotropic subspaces of $V^{2 n}$. We denote this poset by $\mathcal{S}_{n}$.

Let $\left\{e_{i}\right\}_{1 \leq i \leq 2 n}$ be a basis for $V^{2 n}$. It decomposes as the orthogonal direct sum of 2-dimensional subspaces

$$
V^{2 n}=\bigoplus_{i=1}^{n}\left\langle e_{2 i-1}, e_{2 i}\right\rangle,
$$

where

$$
\left(e_{i}, e_{j}\right)= \begin{cases}1 & \text { if }\{j, k\}=\{2 i-1,2 i\} \\ 0 & \text { otherwise }\end{cases}
$$


For an abelian subgroup $A$ of $G_{n}$, the assignment $A \mapsto \pi(A)$ defines a surjective map of posets $\mathcal{A}\left(G_{n}\right) \rightarrow \mathcal{S}_{n}$ which induces an isomorphism

$$
\operatorname{colim}_{\mathcal{A}\left(G_{n}\right)} A / Z\left(G_{n}\right) \rightarrow \operatorname{colim}_{\mathcal{S}_{n}} S .
$$

Note that we use $\mathcal{S}_{n}$ as an indexing category, the colimit is still in the category of groups.

Let us introduce some notation. We denote the multiplication in the image of $\iota_{S}: S \rightarrow$ $\operatorname{colim}_{\mathcal{S}_{n}} S$ by $\iota_{S}\left(s_{1}+s_{2}\right)=\iota_{S}\left(s_{1}\right) \iota_{S}\left(s_{2}\right)$. In particular, for $S=\left\langle e_{I}\right\rangle$, where $e_{I}=$ $\sum_{i \in I} e_{i}$ for some non-empty subset $I$ of $\{1,2, \ldots, 2 n\}$, we simply write

$$
g_{I}=\iota_{\left\langle e_{I}\right\rangle}\left(e_{I}\right)
$$

to denote the image.

Let $\mathcal{S}_{n, r}$ denote the subposet of $\mathcal{S}_{n}$ which consists of subspaces of dimension at most $r$. Theorem 6.1 implies that the inclusion $\mathcal{S}_{n, 2} \rightarrow \mathcal{S}_{n}$ induces an isomorphism

$$
\operatorname{colim}_{\mathcal{S}_{n, 2}} S \rightarrow \operatorname{colim}_{\mathcal{S}_{n}} S
$$

Then a presentation of the colimit can be given as

$$
\operatorname{colim}_{\mathcal{S}_{n}} S=\left\langle g_{I}, I \subset\{1,2, \ldots, 2 n\} \mid \begin{array}{r}
g_{I}^{2}=1,\left[g_{I}, g_{J}\right]=1 \Leftrightarrow\left(e_{I}, e_{J}\right)=0, \\
g_{I} g_{J}=g_{K} \Leftrightarrow e_{I}+e_{J}=e_{K}
\end{array}\right\rangle .
$$

Note that this group is generated by the images $g_{I}=\iota_{\left\langle e_{I}\right\rangle}\left(e_{I}\right)$ of 1-dimensional subspaces $\left\langle e_{I}\right\rangle \in \mathcal{S}_{n, 1}$. We will find a smaller collection of subspaces which generates this group.

For a 1 -dimensional subspace $\left\langle e_{I}\right\rangle \in \mathcal{S}_{n, 1}$, define

$$
\mathcal{L}\left(e_{I}\right)=\left\{W \in \mathcal{S}_{n, 2} \mid \operatorname{dim}(W)=2 \text { and } e_{I} \in W\right\}
$$

and for a sub-collection $\mathcal{T} \subset \mathcal{S}_{n, 2}$ of 2-dimensional spaces let

$$
\mathcal{P}(\mathcal{T})=\left\{\langle w\rangle \in \mathcal{S}_{n, 1} \mid w \subset X \text { for some } X \in \mathcal{T}\right\} .
$$

It is possible to determine the cardinalities of these sets: $\left|\mathcal{L}\left(e_{I}\right)\right|=2^{n}-1$ and $|\mathcal{P}(\{W\})|=3$ for any 2-dimensional subspace $W \in \mathcal{S}_{n, 2}$.

Definition 7.2 Let $\mathcal{Q} \subset \mathcal{S}_{n, 1}$ and $\langle w\rangle \in \mathcal{S}_{n, 1}$, we say $\langle w\rangle$ is connected to $\mathcal{Q}$ if there exists a $W \in \mathcal{S}_{n, 2}$ of dimension 2 such that $w \in W$ and $\mathcal{P}(\{W\})-\{\langle w\rangle\} \subset \mathcal{Q}$. We also say $\langle w\rangle \subset W$ is connected to $\mathcal{Q}$ if the other two elements in $\mathcal{P}(\{W\})-\{\langle w\rangle\}$ are connected to $\mathcal{Q}$. 
Lemma 7.3 Consider the poset $\mathcal{P}_{1}=\mathcal{P}\left(\mathcal{L}\left(e_{1}\right)\right) \cup\left\{\left\langle e_{2}\right\rangle\right\}$, then every element of $\mathcal{S}_{n, 1}$ is connected to $\mathcal{P}_{1}$.

Proof Identify the polar space of $V^{2 n} /\left\langle e_{1}, e_{2}\right\rangle$ with $\mathcal{S}_{n-1}$ and fix $I=\{1,2\}$. Then by (7.1.1) we have

$$
\begin{aligned}
& \mathcal{P}\left(\mathcal{L}\left(e_{1}\right)\right)=\mathcal{S}_{n-1,1} \sqcup\left\{\left\langle e_{1}\right\rangle\right\} \sqcup e_{1} \mathcal{S}_{n-1,1}, \\
& \mathcal{P}\left(\mathcal{L}\left(e_{2}\right)\right)=\mathcal{S}_{n-1,1} \sqcup\left\{\left\langle e_{2}\right\rangle\right\} \sqcup e_{2} \mathcal{S}_{n-1,1}, \\
& \mathcal{P}\left(\mathcal{L}\left(e_{I}\right)\right)=\mathcal{S}_{n-1,1} \sqcup\left\{\left\langle e_{I}\right\rangle\right\} \sqcup e_{I} \mathcal{S}_{n-1,1},
\end{aligned}
$$

where the notation $w \mathcal{S}_{n-1,1}$ means the set $\left\{\langle w+v\rangle \mid\langle v\rangle \in \mathcal{S}_{n-1,1}\right\}$. Also observe that

$$
\mathcal{S}_{n, 1}=\mathcal{P}\left(\mathcal{L}\left(e_{1}\right)\right) \cup \mathcal{P}\left(\mathcal{L}\left(e_{2}\right)\right) \cup \mathcal{P}\left(\mathcal{L}\left(e_{I}\right)\right) .
$$

Every element $\langle v\rangle$ of $e_{2} \mathcal{S}_{n-1,1}$ is connected to $\mathcal{P}_{1}$ via the space $\left\langle e_{2}, v\right\rangle$. Therefore every element of $\mathcal{P}\left(\mathcal{L}\left(e_{2}\right)\right)$ is connected to $\mathcal{P}_{1}$.

To see that every element of $\mathcal{P}\left(\mathcal{L}\left(e_{I}\right)\right)$ is connected to $\mathcal{P}_{1}$, it is sufficient to show that $\left\langle e_{I}\right\rangle$ is connected to $\mathcal{P}_{1}$. The sequence of spaces

$$
\left\langle e_{1}+e_{2}, e_{3}+e_{4}\right\rangle \hookleftarrow\left\langle e_{1}+e_{2}+e_{3}+e_{4}\right\rangle \hookrightarrow\left\langle e_{1}+e_{3}, e_{2}+e_{4}\right\rangle
$$

connects $e_{I}$ to $\mathcal{P}_{1}$.

Set $K_{n}=\operatorname{colim}_{\mathcal{S}_{n, 2}} S$ and consider the map $\phi: \operatorname{colim}_{\mathcal{P}_{1}} S \rightarrow K_{n}$ induced by the inclusion $\mathcal{P}_{1} \rightarrow \mathcal{S}_{n, 2}$. The lemma translates into the statement that the image of $\phi$ generates $K_{n}$. This follows from the definition of connectivity: An element $\left\langle e_{I}\right\rangle$ of $\mathcal{S}_{n, 1}$ is connected to $\mathcal{P}_{1}$ translates into $g_{I}$ lies in the image of $\phi$. Hence an equivalent statement is the following.

Lemma 7.4 Inclusion of the poset $\mathcal{P}_{1} \rightarrow \mathcal{S}_{n, 2}$ induces a surjective map on the colimits

$$
\phi: \operatorname{colim}_{\mathcal{P}_{1}} S \rightarrow K_{n}
$$

By definition, $g_{1}=\iota_{\left\langle e_{1}\right\rangle}\left(e_{1}\right)$ commutes with the generators coming from $\mathcal{P}\left(\mathcal{L}\left(e_{1}\right)\right)$. We will prove that in fact $g_{1}$ also commutes with $g_{2}$ the generator corresponding to $e_{2}$, that is $g_{1}$ commutes with the generators $\mathcal{P}_{1}=\mathcal{P}\left(\mathcal{L}\left(e_{1}\right)\right) \cup\left\{\left\langle e_{2}\right\rangle\right\}$ of the group $K_{n}$. Hence $g_{1}$ is in the center $Z\left(K_{n}\right)$.

Lemma 7.5 For $n \geq 2$

$$
\operatorname{colim}_{\mathcal{A}\left(G_{n}\right)} A / Z\left(G_{n}\right) \cong(\mathbb{Z} / 2)^{2 n+1} .
$$


Proof First recall from (7.1.2) that each generator of $K_{n}$ has order 2, showing that it is also abelian is sufficient. We do induction on $n$. First, we compute the case $n=2$. By the decomposition (7.1.1), it is easy to see

$$
\mathcal{P}\left(\mathcal{L}\left(e_{1}\right)\right)=\left\{\left\langle e_{1}\right\rangle,\left\langle e_{3}\right\rangle,\left\langle e_{1}+e_{3}\right\rangle,\left\langle e_{4}\right\rangle,\left\langle e_{1}+e_{4}\right\rangle,\left\langle e_{3}+e_{4}\right\rangle,\left\langle e_{1}+e_{3}+e_{4}\right\rangle\right\} .
$$

One can check that the minimal number of generators of the image of $\phi$, in Lemma 7.4, is 5 and those are $g_{1}, g_{3}, g_{4}, g_{3,4}$ and $g_{2}$. Observe that $g_{1}$ commutes with all but $g_{2}$. We have the following relations in $K_{2}$ :

$$
\begin{aligned}
g_{2} g_{2,3,4} & =g_{1} g_{1,3,4} & & g_{2} g_{1,3} g_{1,2,4}=g_{1} g_{1,3,4} \\
g_{2} g_{1,3} g_{2,3} g_{1,3,4} & =g_{1} g_{1,3,4} & & g_{2} g_{1} g_{3} g_{2} g_{3}=g_{1} \\
g_{2} g_{1} g_{2} & =g_{1} & &
\end{aligned}
$$

Therefore $g_{1}$ is in the center of $K_{2}$. It suffices to show that the remaining generators in $J=\left\{g_{1}, g_{3}, g_{4}, g_{3,4}, g_{2}\right\}$ are also central. Given $g$ in $J$ there exists an automorphism of $(\mathbb{Z} / 2)^{4}$ which induces an automorphism on $K_{2}$ such that $g$ is mapped to $g_{1}$. Hence any element in $J$ is central in $K_{2}$. We conclude that $K_{2}$ is an abelian group since it is generated by the elements in $J$.

For the general case, first identify the polar space of $V^{2 n} /\left\langle e_{1}, e_{2}\right\rangle$ with $\mathcal{S}_{n-1}$ and by induction the associated colimit is $K_{n-1} \cong(\mathbb{Z} / 2)^{2 n-1}$. Consider $K_{n-1}$ as a subgroup of $K_{n}$. By Lemma 7.4, $K_{n}$ has $2 n+1$ generators, namely $e_{1}, e_{2}$, and the $2 n-1$ generators of $K_{n-1}$. Note that $g_{1}$ and $g_{2}$ centralizes $K_{n-1}$ and the relations (7.5.1) hold in $K_{n}$. Hence $\left[g_{1}, g_{2}\right]=1$.

Recall that the fundamental group of $B\left(2, G_{n}\right)$ is isomorphic to the colimit of abelian subgroups of $G_{n}$.

Theorem 7.6 Let $G_{n}$ denote an extraspecial 2-group of order $2^{2 n+1}$ then

$$
\pi_{1}\left(B\left(2, G_{n}\right)\right) \cong \operatorname{colim}_{\mathcal{N}\left(2, G_{n}\right)} A \cong G_{n} \times \mathbb{Z} / 2 \quad \text { for } n \geq 2 .
$$

Proof Let us denote the colimit in the theorem by $\widehat{K}_{n}$, recall the exact sequence of (7.0.1). There are exact sequences of groups

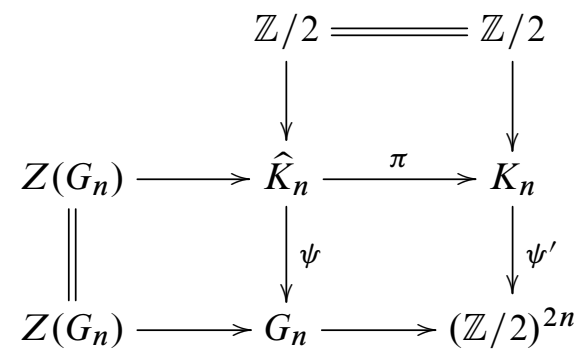


where $Z\left(G_{n}\right) \cong \mathbb{Z} / 2$ and by Lemma $7.5, K_{n} \cong(\mathbb{Z} / 2)^{2 n+1}$ which forces $\operatorname{ker}(\psi) \cong \mathbb{Z} / 2$. Note that $Z\left(G_{n}\right) \cap \operatorname{ker}(\psi)=1$ which implies that this kernel is in the center $Z\left(\widehat{K}_{n}\right)$, and $\psi$ induces an isomorphism

$$
\left[\widehat{K}_{n}, \widehat{K}_{n}\right] \cong\left[G_{n}, G_{n}\right] .
$$

This implies that $\left[\widehat{K}_{n}, \widehat{K}_{n}\right] \cap \operatorname{ker}(\psi)=1$ and $\left[\widehat{K}_{n}, \widehat{K}_{n}\right]=Z\left(G_{n}\right)$.

Denote the product $\left[\widehat{K}_{n}, \widehat{K}_{n}\right] \operatorname{ker}(\psi)$ in $\widehat{K}_{n}$ by $V$. Choose a complement $\bar{C}$ of $V /\left[\widehat{K}_{n}, \widehat{K}_{n}\right]$ in $\hat{K}_{n} /\left[\widehat{K}_{n}, \widehat{K}_{n}\right]$. Let $C$ be the corresponding subgroup of $\hat{K}_{n}$. Then $\widehat{K}_{n}=C \operatorname{ker}(\psi)$ and $C \cap \operatorname{ker}(\psi)=1$, hence

$$
C \cong \widehat{K}_{n} / \operatorname{ker}(\psi) \cong G_{n}
$$

This implies that $\hat{K}_{n}$ is isomorphic to the direct product $G_{n} \times \mathbb{Z} / 2$.

\section{Examples}

\subsection{Transitively commutative groups}

A non-abelian group $G$ is called a transitively commutative group (TC-group) if commutation is a transitive relation for non-central elements that is $[x, y]=1=[y, z]$ implies $[x, z]=1$ for all $x, y, z \in G-Z(G)$. Any pair of maximal abelian subgroups of $G$ intersect at the center $Z(G)$.

For TC-groups, the homotopy types of $E(2, G)$ and $B(2, G)$ are studied by Adem [1]. The exact sequence (3.5.3) of homotopy groups associated to the fibration $E(2, G) \longrightarrow$ $B(2, G) \longrightarrow B G$ gives

$$
1 \longrightarrow T(2) \longrightarrow G(2) \longrightarrow G \longrightarrow 1,
$$

where $G(2)$ is isomorphic to the amalgamated product of maximal abelian subgroups $\left\{M_{i}\right\}_{1 \leq i \leq k}$ along the center of the group, and $T(2)$ is a free group of certain rank. It turns out that $E(2, G)$ and $B(2, G)$ are $K(\pi, 1)$ spaces: $E(2, G)$ is homotopy equivalent to a wedge of circles and $B(2, G) \simeq B(G(2))$.

Higher limits of the representation ring functor $R: \mathcal{N}(2, G) \rightarrow \mathbf{A b}$ fit into an exact sequence

$$
0 \longrightarrow \lim ^{0} R \longrightarrow R(Z(G)) \oplus \bigoplus_{i=1}^{k} R\left(M_{i}\right) \stackrel{\theta}{\longrightarrow} \bigoplus_{i=1}^{k} R(Z(G)) \longrightarrow \lim ^{1} R \longrightarrow 0,
$$

where $\theta\left(z, m_{1}, \ldots, m_{k}\right)=\left(z-\operatorname{res}_{M_{1}, Z(G)} m_{1}, \ldots, z-\operatorname{res}_{M_{k}, Z(G)} m_{k}\right)$. No higher limit occurs $\lim ^{1} R=0$, that is $\theta$ is surjective, since the restriction maps are surjective 
$R\left(M_{i}\right) \rightarrow R(Z(G))$. The same conclusion holds for the restriction of the functor $R$ to $\mathcal{N}(2, G)_{p} \subset \mathcal{N}(2, G)$. Therefore the spectral sequence in (5.0.2) collapses at the $E_{2}$-page and after completion

$$
K^{i}(B(2, G)) \cong \begin{cases}\mathbb{Z} \oplus \bigoplus_{p|| G \mid} \mathbb{Z}_{p}^{n_{p}} & \text { if } i=0 \\ 0 & \text { if } i=1 .\end{cases}
$$

\subsection{Extraspecial 2-groups}

This class of groups are interesting since they provide counter examples to the following question.

Question 8.1 (Adem [1, page 15]) If $G$ is a finite group, are the spaces $B(q, G)$ Eilenberg-Mac Lane spaces of type $K(\pi, 1)$ ?

Recall from Section 7 that an extraspecial 2-group of order $2^{2 n+1}$ is an extension of the form

$$
\mathbb{Z} / 2 \longrightarrow G_{n} \stackrel{\pi}{\longrightarrow}(\mathbb{Z} / 2)^{2 n},
$$

where $\mathbb{Z} / 2$ is the center $Z\left(G_{n}\right)$. We consider the homotopy type of $B\left(2, G_{n}\right)$ for this class of groups. There is a fibration $E\left(2, G_{n}\right) \longrightarrow B\left(2, G_{n}\right) \longrightarrow B G_{n}$ and a corresponding exact sequence of homotopy groups (3.5.3)

$$
1 \longrightarrow \pi_{1}\left(E\left(2, G_{n}\right)\right) \longrightarrow \pi_{1}\left(B\left(2, G_{n}\right)\right) \stackrel{\psi}{\longrightarrow} \pi_{1}\left(B G_{n}\right) \longrightarrow 1,
$$

where $\psi$ is the natural map

$$
\pi_{1}\left(B\left(2, G_{n}\right)\right) \cong \operatorname{colim}_{\mathcal{N}\left(2, G_{n}\right)} A \rightarrow G
$$

induced by inclusions of abelian subgroups $A \subseteq G$. We calculated this colimit in Theorem 7.6

$$
\operatorname{colim}_{\mathcal{N}\left(2, G_{n}\right)} A \cong G_{n} \times \mathbb{Z} / 2
$$

which forces the kernel of $\psi$ to be isomorphic to $\mathbb{Z} / 2$. This implies that the fundamental group of $E\left(2, G_{n}\right)$ is isomorphic to $\mathbb{Z} / 2$. Recall the homotopy colimit description of $E(2, G)$, there is a weak equivalence

$$
E\left(2, G_{n}\right) \simeq \operatorname{hocolim}_{\mathcal{N}\left(2, G_{n}\right)} G_{n} / A .
$$

This homotopy colimit is a finite-dimensional complex. It can be described as the nerve of a category with finitely many morphisms. Therefore $H^{*}\left(E\left(2, G_{n}\right) ; \mathbb{Z}\right)$ is non-zero only for finitely many degrees whereas it is well-known that $H^{*}(B \mathbb{Z} / 2 ; \mathbb{Z})$ is non-zero for every even degree. Therefore $E\left(2, G_{n}\right)$ does not have the homotopy type 
of a $K(\mathbb{Z} / 2,1)$ space, that is it has non-vanishing higher homotopy groups. The same conclusion holds for $B\left(2, G_{n}\right)$ since from the fibration $E(2, G) \rightarrow B(2, G) \rightarrow B G$ we see that

$$
\pi_{k}\left(E\left(2, G_{n}\right)\right) \cong \pi_{k}\left(B\left(2, G_{n}\right)\right) \text { for } k \geq 2 .
$$

Thus $B\left(2, G_{n}\right)$ does not have the homotopy type of $K\left(G_{n} \times \mathbb{Z} / 2,1\right)$.

Indeed for the extraspecial 2-groups of order $32, G_{2}=G_{2}^{+}$or $G_{2}^{-}$, consider the fibration

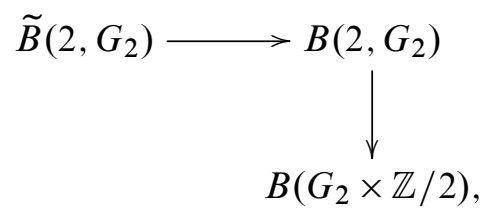

where the fibre is a simply connected complex of dimension 2

$$
\widetilde{B}\left(2, G_{2}\right)=\operatorname{hocolim}_{\mathcal{N}\left(2, G_{2}\right)}\left(G_{2} \times \mathbb{Z} / 2\right) / A .
$$

Cohomology groups of this fibre can be calculated from the chain complex

$$
\prod_{A_{0} \leftarrow A_{1} \leftarrow A_{2}} \mathbb{Z}\left[\left(G_{2} \times \mathbb{Z} / 2\right) / A_{2}\right] \longrightarrow \prod_{A_{0} \leftarrow A_{1}} \mathbb{Z}\left[\left(G_{2} \times \mathbb{Z} / 2\right) / A_{1}\right] \longrightarrow \prod_{A_{0}} \mathbb{Z}\left[\left(G_{2} \times \mathbb{Z} / 2\right) / A_{0}\right]
$$

where $A_{i}$ are abelian subgroups of $G_{n}$. It is sufficient to consider the ones which contain the center $Z\left(G_{n}\right)$ (Remark 3.2). By a counting argument using the structure of the poset which is isomorphic to the polar space (Definition 7.1) of $\mathrm{Sp}_{4}$ (2) (union the trivial space 0), one can calculate the Euler characteristic

$$
\begin{aligned}
\chi\left(\widetilde{B}\left(2, G_{2}\right)\right) & =\frac{64}{2}\left|\left\{0 \subset W_{1} \subset W_{2}\right\}\right|-\frac{64}{2\left|W_{1}\right|}\left|\left\{W_{1} \subset W_{2}\right\}\right|+\frac{64}{2\left|W_{2}\right|}\left|\left\{W_{2}\right\}\right| \\
& =\frac{64}{2} 45-\left(\frac{64}{4} 45+\frac{64}{2} 30\right)+\left(\frac{64}{8} 15+\frac{64}{4} 15+\frac{64}{2}\right)=152,
\end{aligned}
$$

where $W_{i}$ are the isotropic subspaces of $V^{4}=(\mathbb{Z} / 2)^{4}$ as described in Section 7. It follows that the higher homotopy groups of $B\left(2, G_{2}\right)$ are isomorphic to the homotopy groups of a wedge of spheres

$$
\pi_{i}\left(B\left(2, G_{2}\right)\right) \cong \pi_{i}\left(\bigvee^{151} S^{2}\right) \quad \text { for } i>1 .
$$

As for the $K$-theory, non-vanishing higher limits of the representation ring functor occur in the Bousfield-Kan spectral sequence. Again in the case of $G_{2}$ a calculation in GAP [12] shows that

$$
{\underset{\lim }{\longleftarrow}}_{\mathcal{N}\left(2, G_{2}\right)}^{i} R \cong \begin{cases}\mathbb{Z}^{32} & i=0, \\ (\mathbb{Z} / 2)^{9} & i=1, \\ 0 & \text { otherwise }\end{cases}
$$


Therefore the spectral sequence in (5.0.2) collapses at the $E_{2}$-page and after completion

$$
K^{i}\left(B\left(2, G_{2}\right)\right) \cong \begin{cases}\mathbb{Z} \oplus \mathbb{Z}_{2}^{31} & \text { if } i=0 \\ (\mathbb{Z} / 2)^{9} & \text { if } i=1 .\end{cases}
$$

Note that the $K$-theory computation also confirms that $B\left(G_{2}, 2\right)$ is not homotopy equivalent to $B\left(G_{2} \times \mathbb{Z} / 2\right)$, as the latter has $K^{1}\left(B\left(G_{2} \times \mathbb{Z} / 2\right)\right)=0$ as a consequence of the Atiyah-Segal completion theorem.

\section{Appendix A: Simplicial sets}

In this section we review some definitions about simplicial sets especially homotopy colimits. We refer the reader to Goerss [10] and Dwyer [7].

The category of simplicial sets $\mathbf{S}$ and the category of topological spaces Top are closely related. Given $T$ a topological space there is a functor $\mathbf{T o p} \rightarrow \mathbf{S}$ called the singular set defined by

$$
\operatorname{Sing}(T): \mathbf{n} \mapsto \operatorname{Hom}_{\mathbf{T o p}}\left(\left|\Delta^{n}\right|, T\right),
$$

where $\left|\Delta^{n}\right|$ is the topological standard $n$-simplex. Conversely a simplicial set $X$ gives rise to a topological space via the geometric realization functor defined by the difference cokernel

$$
|X|=\lim _{\longrightarrow}\left\{\coprod_{\theta: \mathbf{m} \rightarrow \mathbf{n}} X_{n} \times\left|\Delta^{m}\right| \rightrightarrows \coprod_{\mathbf{n}} X_{n} \times\left|\Delta^{n}\right|\right\},
$$

where $(x, p) \mapsto\left(x, \theta_{*}(p)\right)$ and $(x, p) \mapsto\left(\theta^{*}(x), p\right)$. There is an adjoint relation between these two functors

$$
|\cdot|: \mathbf{S} \rightleftarrows \text { Top :Sing } .
$$

Note that $|\cdot|$ preserves all colimits in $\mathbf{S}$ since it has a right adjoint.

Let $\mathbf{I}$ be a small category, and $i \in \mathbf{I}$ an object, the under category $\mathbf{I} \backslash i$ is the category whose objects are the maps $i \rightarrow i_{0}$ and morphisms from $i \rightarrow i_{0}$ to $i \rightarrow i_{1}$ are commutative diagrams

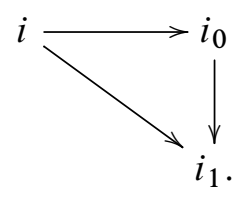


Let $F: \mathbf{I} \rightarrow$ Top be a functor. The homotopy colimit of $F$ is the topological space defined by the difference cokernel

$$
\operatorname{hocolim} F=\underline{\lim }\left\{\coprod_{f: j \rightarrow i} B(\mathbf{I} \backslash i) \times F(j) \rightrightarrows \coprod_{i} B(\mathbf{I} \backslash i) \times F(i)\right\},
$$

where $(b, y) \mapsto(B(f)(b), y)$ and $(b, y) \mapsto(b, f(y))$. If one wants to work in the category of simplicial sets, given $F: \mathbf{I} \rightarrow \mathbf{S}$ one can use the simplicial set $B_{\bullet}(\mathbf{I} \backslash i)$ instead.

From the definition we see that there is a natural map to the ordinary colimit

$$
\text { hocolim } F \rightarrow \operatorname{colim} F
$$

obtained by collapsing the nerves $B(\mathbf{I} \backslash i) \rightarrow$ pt. This map is not usually a weak equivalence. In general, it is a weak equivalence if the diagram $F: \mathbf{I} \rightarrow \mathbf{S}$ is a free diagram (see Farjoun [8, Appendix HC]). A diagram of sets $\mathbf{I} \rightarrow$ Set is free if it is of the form $\bigsqcup_{i} F^{i}$ for a collection of subobjects of $\mathbf{I}$ where each diagram $F^{i}: \mathbf{I} \rightarrow$ Set is defined by $F^{i}(j)=\operatorname{Hom}_{\mathbf{I}}(i, j)$. A diagram of simplicial sets is free if in each dimension it gives a free diagram of sets. In particular, a collection of sets which is closed under taking subsets and partially ordered by inclusion gives a free diagram of sets.

A standard example of a homotopy colimit is the Borel construction. Let $X$ be a $G$-space and consider $\mathbf{G}$ the category associated to the group then

$$
\text { hocolim } F \simeq X \times_{G} E G,
$$

where $F: \mathbf{G} \rightarrow$ Top sends the single object to $X$. The most important property of homotopy colimits is that a natural transformation $F \rightarrow F^{\prime}$ of functors such that $F(i) \rightarrow F^{\prime}(i)$ is a weak equivalence for all $i \in \mathbf{I}$ induces a weak equivalence

$$
\text { hocolim } F \rightarrow \text { hocolim } F^{\prime} \text {. }
$$

Another useful property we use in the paper is the commutativity of homotopy colimits. We refer the reader to Welker, Ziegler and Živaljević [21] for further properties of homotopy colimits.

\section{References}

[1] A Adem, F R Cohen, E Torres Giese, Commuting elements, simplicial spaces and filtrations of classifying spaces, Math. Proc. Cambridge Philos. Soc. 152 (2012) 91-114 MR2860418 
[2] M F Atiyah, G B Segal, Equivariant K-theory and completion, J. Differential Geometry 3 (1969) 1-18 MR0259946

[3] A K Bousfield, D M Kan, Homotopy limits, completions and localizations, Lecture Notes in Mathematics 304, Springer, Berlin (1972) MR0365573

[4] KS Brown, The coset poset and probabilistic zeta function of a finite group, J. Algebra 225 (2000) 989-1012 MR1741574

[5] J D Dixon, M P F du Sautoy, A Mann, D Segal, Analytic pro-p groups, 2nd edition, Cambridge Studies Adv. Math. 61, Cambridge Univ. Press (1999) MR1720368

[6] A W M Dress, Contributions to the theory of induced representations, from: "Algebraic $K$-theory, II: "Classical" algebraic $K$-theory and connections with arithmetic (Proc. Conf., Battelle Memorial Inst.)”, Lecture Notes in Math. 342, Springer, Berlin (1973) 183-240 MR0384917

[7] W G Dwyer, Homology decompositions for classifying spaces of finite groups, Topology 36 (1997) 783-804 MR1432421

[8] E D Farjoun, Cellular spaces, null spaces and homotopy localization, Lecture Notes in Mathematics 1622, Springer, Berlin (1996) MR1392221

[9] E D Farjoun, Fundamental group of homotopy colimits, Adv. Math. 182 (2004) 1-27 MR2028495

[10] P G Goerss, J F Jardine, Simplicial homotopy theory, Progress in Mathematics 174, Birkhäuser, Basel (1999) MR1711612

[11] J Grodal, Higher limits via subgroup complexes, Ann. of Math. 155 (2002) 405-457 MR1906592

[12] T G group, GAP: Groups, algorithms and programming (2008) Available at http:// WwW.gap-system.org/

[13] S Jackowski, J McClure, Homotopy decomposition of classifying spaces via elementary abelian subgroups, Topology 31 (1992) 113-132 MR1153240

[14] C-N Lee, A homotopy decomposition for the classifying space of virtually torsion-free groups and applications, Math. Proc. Cambridge Philos. Soc. 120 (1996) 663-686 MR1401955

[15] W Lück, Rational computations of the topological K-theory of classifying spaces of discrete groups, J. Reine Angew. Math. 611 (2007) 163-187 MR2361088

[16] N Nikolov, D Segal, Generators and commutators in finite groups; abstract quotients of compact groups, Invent. Math. 190 (2012) 513-602 MR2995181

[17] B Oliver, Higher limits via Steinberg representations, Comm. Algebra 22 (1994) 13811393 MR1261265

[18] G Segal, Equivariant K-theory, Inst. Hautes Études Sci. Publ. Math. (1968) 129-151 MR0234452 
[19] S D Smith, Subgroup complexes, Mathematical Surveys and Monographs 179, Amer. Math. Soc. (2011) MR2850680

[20] P Webb, An introduction to the representations and cohomology of categories, from: "Group representation theory", EPFL Press, Lausanne (2007) 149-173 MR2336640

[21] V Welker, G M Ziegler, R T Živaljević, Homotopy colimits: Comparison lemmas for combinatorial applications, J. Reine Angew. Math. 509 (1999) 117-149 MR1679169

Department of Mathematics, The University of British Columbia Vancouver BC V6T 1Z2, Canada

okay@math.ubc.ca

http://www.math.ubc.ca/ okay/

Received: 18 July 2013 Revised: 12 September 2013 
